\title{
RESIDUAL DISCRIMINANT AND RESIDUAL BIFURCATION LOCUS OF A FUNCTION GERM SINGULAR ALONG AN EULER FREE DIVISOR
}

\author{
VINCENT GRANDJEAN
}

We are interested in a complex analytic function germ $f$ defined in $\left(\boldsymbol{C}^{n}, 0\right)$ singular along a complete intersection germ $(H, 0)$ of positive dimension, and with singularities more complicated than those of $H$ only at the origin of $\boldsymbol{C}^{n}$ (that is, $f$ is of finite relative codimension on the right along $H$ ). We know ([Pe1]) that such a function germ $f$ admits a mini-versal unfolding $F$. We would like to have informations about the objects which can control the analytic types of deformed function germs arising from $f$ with singularities along $H$.

In the classical setting of isolated complete intersection singularities, it is well known that the discriminant of its mini-versal unfolding is a free divisor (see [Sa1] for hypersurfaces and [Lo] in the general case) and that the logarithmic stratification of this discriminant controls the analytic types of the deformations arising from $f$ (see [Wi], [Sal] for hypersurfaces and [Ti] in the general case). It is also known that the vector fields tangent to this discriminant are liftable and we know how to produce a basis of such tangent vector fields (see [Sa2], [Bru1] or [Ter1] for hypersurfaces and [Go] for the general case). The same kind of results are also true for the bifurcation set of the mini-versal unfolding (see [Ter2] and [Bru2] and $[\mathrm{Go}])$.

Despite the existence of the right objects for these questions in the nonisolated case, that is, $R C_{F}$ the residual critical locus, $R D_{F}$ the residual discriminant and $R B_{F}$ the residual bifurcation locus introduced by Pellikaan [Pe2], as far as the author knows, there are no such results in the case of hypersurface germ of finite codimension on the right along a variety of positive dimension.

Such theorems for any complete intersection germ $H$ (CI for short) of positive dimension, as given singular set, are not easy to find.

In the first section we recall some notations and basic facts about nonisolated hypersurfaces singularities and state a theorem which enables to compute an equation for the residual discriminant when this last one is a hypersurface. In the second section, the main part of this paper, we suppose that $H$ is a hypersurface and we show that such nonisolated singularities are closely related in

1991 Mathematics Subject Classification: Primary 32S05, 32S30, 58K60; Secondary 32B10, 32S60, $58 \mathrm{~K} 40$.

Received October 2, 2000; revised May 1, 2001. 
some way with function germs with isolated singularities on $H$ (see [BR]). We suppose that $H$ is a free divisor and is Euler (see Section 2.3 or [All, p. 2]). With a ringed structure over the residual critical locus $R C_{F}$ different from that given by Pellikaan [Pe2] and under the hypothesis that $\operatorname{dim} R C_{G} \cap\left(H \times C^{p-1}\right)=p-2$ (where $G$ is the truncated mini-versal unfolding associated with $F$ and $p$ is the dimension of the parameter space of the mini-versal unfolding $F$ ), we show that it can be endowed with a reduced complete intersection structure of dimension $p$. By means of Fitting ideals, the residual discriminant $R D_{F}$ becomes a reduced free divisor. Moreover we show that the vector fields tangent to $R D_{F}$ are liftable by $\tilde{F}$ (the unfolding mapping associated with $F$ ). The same results are also true for the residual bifurcation locus $R B_{F}$.

In the last section, thanks to the lists of simple singularities given by Zaharia ([Za1], [Za2]) and when $H$ is a smooth space of codimension 2, we exhibit a family of finite relative codimension on the right along $H$ (which is not a suspension of any function germ singular along a smooth hypersurface) such that the residual discriminant of any function germ of this kind is a complete intersection of codimension 2 .

\section{Residual critical locus and residual discriminant, first facts}

\subsection{Introduction}

Let $x:=\left(x_{1}, \ldots, x_{n}\right)$ denote coordinates at the origin of $\boldsymbol{C}^{n}$ and $y$ a coordinate at $0 \in \boldsymbol{C}$. Let $\mathcal{O}_{x}$ denote the local $\boldsymbol{C}$-algebra of holomorphic function germs at the origin of $\boldsymbol{C}^{n}$. Let $I$ be the reduced ideal of a complete intersection germ $(H, 0) \subset\left(\boldsymbol{C}^{n}, 0\right)$. We recall that $\mathscr{R}_{I}$ is the subgroup of the automorphisms of $\mathcal{O}_{x}$ which preserve the ideal $I$ and $\mathscr{R}_{I, e}$ is the subgroup of the biholomorphisms which preserve the ideal $I$ (the target may move), and they act naturally on $\mathcal{O}_{x}$ and $I$. We also recall that $\mathscr{K}_{I, e}$ denotes the subgroup of the extended contact group $\mathscr{K}_{e}$ which preserves the ideal $I$.

If $z$ denotes any system of coordinates at a point 0 of a smooth space (germ) $\left(C^{l}, 0\right)(l \geq 1)$, we denote by $\Theta_{z}$ the $\mathcal{O}_{z}$-free module of rank $l$ of smooth vector field germs on $\left(\boldsymbol{C}^{l}, 0\right)$. If $X$ is an analytic set germ in $\left(\boldsymbol{C}^{l}, 0\right)$ defined by a reduced ideal $J$, then we denote by $\Theta_{X}$ or $\Theta_{J, e}$ the $\mathcal{O}_{z}$-module of the vector fields tangent to $X$, see [Pe1].

Let $f \in I^{2}$, which is equal to the primitive ideal associated with $I$, i.e., the ideal of the holomorphic function germs vanishing over $H$ and with their first derivatives vanishing over $H$ (see [Pe1]). We assume that $f$ is $\mathscr{R}_{I}$-finitely determined (or of finite relative codimension on the right along $H$ or just of finite relative codimension along $H$ when the context is clear). This means that the complex vector space $I^{2} / T_{e} \mathscr{R}_{I}(f)$ is finite dimensional, with $T_{e} \mathscr{R}_{I}(f):=$ $\left\{\xi \cdot f: \xi \in \Theta_{I, e}\right\}$.

Remark 1.1. Let $f$ be of finite relative codimension along $H$, and let us denote by $(d f)$ the Jacobian ideal of $f$. Then $V\left(T_{e} \mathscr{R}_{I}(f)\right)=V((d f))$. 
Let $F(x, u):=f(x)+\sum_{k=1}^{p} u_{k} e_{k}(x)$ be a mini-versal unfolding of $f$, where $p$ is the dimension of the complex vector space $I^{2} / T_{e} \mathscr{R}_{I}(f)$ and $\left\{e_{1}, \ldots, e_{p}\right\}$ is a basis of this vector space. Let us denote by $\tilde{I}:=I \otimes_{\mathcal{O}_{x}} \mathcal{O}_{x, u}$ and by $\tilde{\Theta}_{I, e}:=$ $\Theta_{I, e} \otimes_{\mathcal{O}_{x}} \mathcal{O}_{x, u}$ the $\mathcal{O}_{x, u}$-module of the vertical vector fields tangent to $\left(H \times \boldsymbol{C}^{p}, 0\right)$ (in the following the parameter space $\left(\boldsymbol{C}^{p}, 0\right)$ can be seen as the horizontal space) and by $\tilde{T}_{e} \mathscr{R}_{I}(f)$ the ideal in $\mathcal{O}_{x, u}$ generated by the $\tilde{\xi} \cdot F$ 's, where $\tilde{\xi} \in \tilde{\Theta}_{I, e}$. Then by the Malgrange-Weierstrass Preparation Theorem, the $\mathcal{O}_{x, u}$-module $\tilde{I}^{2} / \tilde{T}_{e} \mathscr{R}_{I}(f)$ is an $\mathcal{O}_{u}$-module finitely generated by $e_{1}, \ldots, e_{p}$. Let $f_{u}(x):=F(x, u)$. We want to investigate where the $f_{u}$ 's present at the origin some singularities more complicated than the singularities of $H$. For this purpose we introduce the following definitions.

Definition 1.1 ([Pe2, Section 2] and [Ji, Chapter 2.2]). The support in $\left(\boldsymbol{C}^{n} \times \boldsymbol{C}^{p}, 0\right)$ of the analytic coherent sheaf generated by $\tilde{I}^{2} / \tilde{T}_{e} \mathscr{R}_{I}(f)$ is denoted by $R C_{F}$ and is called the residual critical locus of $F$. We call $\tilde{I}^{2} / \tilde{T}_{e} \mathscr{R}_{I}(f)$ the residual Jacobian module of $F$ and denote it by $M_{R C_{F}}$. The image $R D_{F}$ of $R C_{F}$ by $F \times i d_{\left(C^{p}, 0\right)}$ is called the residual discriminant of $F$.

Our definitions use some ringed structure slightly different from those of Pellikaan and Jiang, who are particulary interested in morsification of a germ $f$ (with one-dimensional singular set, in order to find the expected numbers of the generic transversal singularities), but these differences do not change the geometric properties of these zero loci.

Let $\tilde{F}:=F \times i d_{\left(C^{p}, 0\right)}:\left(\boldsymbol{C}^{n} \times \boldsymbol{C}^{p}, 0\right) \rightarrow\left(\boldsymbol{C} \times \boldsymbol{C}^{p}, 0\right)$ be the unfolding mapping associated with the mini-versal unfolding of $f$, and let $\pi$ be the projection on the second factor $\left(\boldsymbol{C}^{p}, 0\right)$.

Now let us recall what the 0-Fitting ideal of a module is. Let $M$ be any finitely generated $A$-module of finite presentation. Then there is an exact sequence

$$
A^{p} \stackrel{\Psi}{\rightarrow} A^{q} \rightarrow M \rightarrow 0 .
$$

The 0 -th Fitting ideal of $M$, denoted by $\mathscr{F}_{0}(M)$, is the ideal generated in $A$ by the $q \times q$ minors of the matrix of the linear mapping $\Psi: A^{p} \rightarrow A^{q}$. This ideal does not depend on the presentation and behaves well under base change (see [To, I.2] and [Tei, Section 1]) and is such that $\mathscr{F}_{0}(M) \subset A n n_{A}(M)$ and $\sqrt{\mathscr{F}_{0}(M)}=$ $\sqrt{A n n_{A}(M)}$, which is a key point, since it enables to provide some analytic ringed structure (non reduced in general) to images of finite mappings (see [Tei]).

LEMMA 1.2. With the above hypotheses, the following restriction germs are finite maps

$$
\left.\tilde{F}\right|_{R C_{F}}: R C_{F} \rightarrow\left(C^{p} \times C, 0\right) \text { and }\left.\pi\right|_{R C_{F}}: R C_{F} \rightarrow\left(\boldsymbol{C}^{p}, 0\right) .
$$

Proof. When $R C_{F}$ is endowed with the following ringed structure $\mathcal{O}_{x, u} / \mathscr{F}_{0}\left(M_{R C_{F}}\right)\left(\right.$ since $\mathcal{O}_{x} / \mathscr{F}_{0}\left(I^{2} / T_{e} \mathscr{R}_{I}(f)\right)$ is a finite dimensional complex vector 
space, it is a finitely generated $\mathcal{O}_{u}$-module), so [Gu, Theorem 5] tells us that we are in presence of a finite map.

Since $\left.\tilde{F}\right|_{R C_{F}}: R C_{F} \rightarrow\left(C^{p} \times C, 0\right)$ is a finite map, $R D_{F}$ is the germ of an analytic space in $\left(\boldsymbol{C}^{p} \times \boldsymbol{C}, 0\right)$ defined (for instance) by the ideal $\mathscr{F}_{0}\left(\tilde{F}_{*}\left(M_{R C_{F}}\right)\right)$ in $\mathcal{O}_{y, u}$, where $\mathscr{F}_{0}$ is the 0 -th Fitting ideal of the given module. Note that the dimension of $R C_{F}$ is always smaller than or equal to $p$.

Remark 1.2. The modules sheaves generated by $M_{R C_{F}}$, denoted by $\mathscr{M}_{R C_{F}}$, and respectively by $\mathcal{O}_{x, u} /\left(d_{x} F\right)$, denoted by $\tilde{\mathcal{O}}_{\Sigma(F)}$, coincide on the open set $\left(\boldsymbol{C}^{n} \times \boldsymbol{C}^{p}, 0\right) \backslash\left(H \times \boldsymbol{C}^{p}, 0\right)$, where $\left(d_{x} F\right)$ is the ideal generated by $\partial F / \partial x_{1}, \ldots$, $\partial F / \partial x_{n}$.

DeFINITION 1.3. We denote by $R B_{F}$ the discriminant locus of the finite analytic mapping $\left.\pi\right|_{R D_{F}}:\left(R D_{F}, 0\right) \rightarrow\left(C^{p}, 0\right)$ and $R B_{F}$ is called the residual bifurcation locus of $F$, where $\pi$ is the projection $(y, u) \rightarrow u$.

\subsection{Providing an equation for the discriminant}

The next result is a reformulation of [ $\mathrm{dPGW}$, Theorem 3.1] in more general terms, which will be very useful when we want to find some equations of the residual discriminant and to lift the vector fields tangent to the residual discriminant.

Let $M=N / L$ be a given $\mathcal{O}_{x, u}$-module of finite type where $N$ and $L$ are some finitely generated submodules (or ideals) of $\mathcal{O}_{x, u}^{l}$ for a positive integer $l$. Let $\mathscr{S}_{M}$ denote its support in $\left(\boldsymbol{C}^{n} \times \boldsymbol{C}^{p}, 0\right)$. Let $\tilde{G}=(G(x, u), u)$ be a holomorphic mapping with $G \in \mathcal{O}_{x, u}$. Let $\pi$ be the projection on $\left(\boldsymbol{C}^{p}, 0\right)$. Note that if $M$ is a free $\mathcal{O}_{u}$-module then $\left.\tilde{G}\right|_{\mathscr{S}_{M}}$ and $\left.\pi\right|_{\mathscr{S}_{M}}$ are finite mappings.

THEOREM 1.4. Suppose that $M$ is a free $\mathcal{O}_{u}$-module of rank $s$. If $\alpha_{1}, \ldots, \alpha_{s} \in$ $\mathcal{O}_{x, u}$ project to a $\boldsymbol{C}$-basis of $M /(\pi \circ \tilde{G})^{*} m_{u} M$, then for each $j=1, \ldots, s, G \alpha_{j}$ is uniquely written as a $(\pi \circ \tilde{G})^{*} \mathcal{O}_{u}$-linear combination in $\alpha_{1}, \ldots, \alpha_{s}$ say

$$
G \alpha_{j}=\sum_{i=1}^{s}(\pi \circ \tilde{G})^{*} a_{i, j} \cdot \alpha_{i} .
$$

Let $A:=\left[a_{i, j}\right]$ and let $\delta:=I_{s}-A$. Then the following sequence is exact

$$
0 \rightarrow \mathcal{O}_{y, u}^{s} \stackrel{\delta}{\rightarrow} \mathcal{O}_{y, u}^{s} \stackrel{\pi}{\rightarrow} M \rightarrow 0
$$

where $\pi\left(e_{i}\right)$ is the projection of the $i$-th basis vector of $\mathcal{O}_{x, u}^{s}$. Moreover the ideal $\langle\operatorname{det} \delta\rangle$ of $\mathcal{O}_{y, u}$ defines the image $\left.\tilde{G}\right|_{\mathscr{S}_{M}}\left(\mathscr{S}_{M}\right)$.

Proof. We claim that the module of the $\mathcal{O}_{y, u}$-relations amongst the $\alpha_{i}$ 's 
is generated by the $\left(r_{j}\right)$ 's. Let $\operatorname{Rel}:=\left\{\left(\phi_{1}, \ldots, \phi_{s}\right) \in \mathcal{O}_{y, u}^{s}: \sum_{j=1}^{s}\left(\phi_{j} \circ \tilde{G}\right) \alpha_{j}=0\right\}$. Let $R_{j}=y e_{j}-\sum_{i=1}^{s} a_{i, j} e_{i}$ then $R e l=\left(R_{1}, \ldots R_{s}\right) \mathcal{O}_{y, u}$. To see this, let $\Phi=$ $\left(\phi_{1}, \ldots, \phi_{s}\right) \in$ Rel. For $j=1, \ldots, s$, we can decompose $\phi_{j}=\beta_{j}+y \gamma_{j}$, where $\beta_{j} \in \mathcal{O}_{u}$ and $\gamma_{j} \in \mathcal{O}_{y, u}$. Thus for each $j$

$$
\tilde{G}^{*} \gamma_{j} \cdot \alpha_{j}=\sum_{i=1}^{s}(\pi \circ \tilde{G})^{*} b_{i, j} \cdot \alpha_{i}
$$

with $b_{i, j} \in \mathcal{O}_{u}$. Let $S_{j}=\gamma_{j} e_{j}-\sum_{i=1}^{s} b_{i, j} e_{i}$, then we have $S_{j} \in R e l$.

$$
\begin{aligned}
\Phi & =\sum_{j=1}^{s}\left(\beta_{j}+y \gamma_{j}\right) e_{j}=\sum_{j=1}^{s} \beta_{j} e_{j}+y \sum_{j=1}^{s}\left(S_{j}+\sum_{i=1}^{s} b_{i, j} e_{i}\right) \\
& =\sum_{j=1}^{s} \beta_{j} e_{j}+y \sum_{j=1}^{s} S_{j}+\sum_{j=1}^{s} \sum_{i=1}^{s} b_{i, j}\left(R_{i}+\sum_{k=1}^{s} a_{i, k} e_{k}\right) \\
& =\sum_{k=1}^{s}\left(\beta_{k}+\sum_{j=1}^{s} \sum_{i=1}^{s} b_{i, j} a_{i, k}\right) e_{k}+y \sum_{j=1}^{s} S_{j}+\sum_{j=1}^{s} \sum_{i=1}^{s} b_{i, j} R_{i} .
\end{aligned}
$$

Since $R_{j}, S_{j}$, and $\Phi$ are in $R e l$ we obtain that

$$
\sum_{k=1}^{s}(\pi \circ \tilde{G})^{*} \theta_{k} \cdot \alpha_{k}=0
$$

where $\theta_{k}=\beta_{k}+\sum_{j=1}^{s} \sum_{i=1}^{s} b_{i, j} a_{i, k} \in \mathcal{O}_{u}$. Since the $\alpha_{k}$ 's form an $\mathcal{O}_{u}$-free basis of $M$, we deduce that $\theta_{k}=0$ for any $k$. Then $\Phi \in m_{y, u} R e l+\left(R_{1}, \ldots, R_{s}\right) \mathcal{O}_{y, u}$ and thus

$$
R e l \subset m_{y, u} \operatorname{Rel}+\left\langle R_{1}, \ldots, R_{s}\right\rangle \mathcal{O}_{y, u},
$$

and then by Nakayama's Lemma $R e l$ is a $\mathcal{O}_{y, u}$-module (as module sheaf, we see that $R e l$ is coherent), finitely generated by $R_{1}, \ldots, R_{s}$. Then the sequence of $\mathcal{O}_{y, u}$-modules

$$
\mathcal{O}_{y, u}^{s} \stackrel{\delta}{\rightarrow} \mathcal{O}_{y, u}^{s} \stackrel{\pi}{\rightarrow} M_{R C_{F}} \rightarrow 0
$$

is exact with $\delta b_{j}=A_{j}$.

If $\delta$ is not injective, there exists a vector $\theta \neq 0$ in $\operatorname{ker} \delta$. Thus there is a Zariski (analytic) open set on which $\theta$ never vanishes. Then $\operatorname{det} \delta$ vanishes over an open set, but this is a contradiction, since $\operatorname{det} \delta$ is a Weierstrass polynomial in $y$ of degree $s$ with coefficients in $\mathcal{O}_{u}$.

Remark 1.3. The previous theorem applies when the depth of $M_{R C_{F}}$ is equal to $p$ and with $\tilde{G}=\tilde{F}$, in which case it is a free $\mathcal{O}_{u}$-module of rank $p$. So $R D_{F}$ is a hypersurface. 


\section{The hypersurface case}

When the ideal $I$ is principal $(I=(h))$, i.e., when we consider functions singular along a (reduced) hypersurface $H:=\{h=0\}$, we can say more in this case than in the general case above.

\subsection{Hypersurfaces singular along a given hypersurface $H$ and hypersurfaces with isolated singularities along $H$}

Arnol'd, in [Ar], studied a function having isolated singularities along a smooth hypersurface (the boundary). This was generalized by [Ly1] when the boundary is an isolated hypersurface singularity. They have given classifications of such isolated singularities and connected them to some groups generated by reflections. They give also some lists of such simple singularities. The general work dealing with functions having isolated singularities on a variety has been done by Bruce and Roberts in $[\mathrm{BR}]$. They study a function $f_{0}$ with an isolated singularity on an analytic set germ $H$, defined by a reduced ideal $I$, and they define a notion of right finite determinacy for these function germs. They give necessary and sufficient (numerical) conditions to be of finite determinacy (on the right) in that case, which is equivalent to the finiteness of $\operatorname{dim}_{C}\left(\mathcal{O}_{x} /\left(\xi_{1} \cdot f_{0}, \ldots, \xi_{t} \cdot f_{0}\right)\right)$, where $\xi_{1}, \ldots, \xi_{t}(t \geq n)$ denote a minimal generating system of $\Theta_{I, e}$. In that case we say that $f_{0}$ has an isolated singularity along $H$ or that $f_{0}$ is of finite codimension on the right along $H$. This is stronger than just having an isolated singularity at the origin, since it means that outside the origin the hypersurface $f_{0}^{-1}(0)$ is tranverse to the leaves of the foliation given by the vector fields tangent to $H$ (the logarithmic strata of $H$ ). In this context, note that Dimca, in [Di], has given some conditions on any isolated hypersurface singularity $H$ to have simple functions (for functions with isolated singularities on $H)$. In the same way Tibăr has also given conditions for functions with isolated singularities on an analytic germ $H$ to be simple when $H$ is an ICIS or, in some other cases, when $H$ has nonisolated singularities [Ti].

In the sequel we will show that under some additional hypotheses on $H$, every function of finite relative codimension on the right along an hypersurface $H$ comes, in fact, from a function having an isolated singularity on $H$.

Let $f$ be any function in $I^{2}$ of finite relative codimension and let $h$ be a generator of $I$. Then the following composition of $\mathcal{O}_{x}$-modules homomorphisms is onto:

$$
\frac{I^{2}}{T_{e} \mathscr{R}_{I}(f)} \stackrel{p_{0}}{\longrightarrow} \frac{I^{2}}{T_{e} \mathscr{K}_{I}(f)} \stackrel{\delta_{h}}{\longrightarrow} \frac{\mathcal{O}_{x}}{T_{e} \mathscr{K}_{I}\left(f_{0}\right)} \stackrel{p_{1}}{\longrightarrow} \frac{\mathcal{O}_{x}}{T_{e} \mathscr{K}\left(f_{0}\right)} \stackrel{p_{2}}{\longrightarrow} 0,
$$

where $f_{0}$ is the function germ such that $f=h^{2} f_{0}, p_{0}, p_{1}$ and $p_{2}$ are the obvious projection maps and $\delta_{h}$ is the homomorphism which maps $h^{2} \alpha$ to $\alpha$. This means that $f_{0}$ has an isolated singularity at the origin and that a basis of the finite dimensional complex vector space $I^{2} / T_{e} \mathscr{R}_{I}(f)$, say $\left\{h^{2} e_{1}, \ldots, h^{2} e_{p}\right\}$, is mapped onto a generating family of the finite dimensional complex vector space $\mathcal{O}_{x} / T_{e} \mathscr{K}\left(f_{0}\right)$. Then the mini-versal unfolding $F$ of $f$ is of the form $F=h^{2} F_{0}$, 
where $F_{0}(x, u):=f_{0}(x)+\sum_{k=1}^{p} u_{k} e_{k}(x)$ is a $\mathscr{K}_{e}$-versal unfolding of $f_{0}$. We can suppose that $e_{p}(x)=-1$. We have $\xi_{j} \cdot h=h \alpha_{j}$ and $\xi_{j} \cdot F=h^{2} A_{j}$ for $j=1, \ldots, t$. We verify that $A_{j}(x, u)=2 \alpha_{j}(x) F_{0}(x, u)+\xi_{j}(x) \cdot F_{0}(x, u)$. Note that the $\mathcal{O}_{x, u^{-}}$ modules $\left(h^{2}\right) / \tilde{T}_{e} \mathscr{R}_{I}(f)$ and $\mathcal{O}_{x, u} /\left(A_{1}, \ldots, A_{t}\right)$ are canonically isomorphic. The following proposition tells us more about $f_{0}$ than being just an isolated hypersurface singularity.

Proposition 2.1. Let $H$ be a hypersurface defined by a reduced equation $\{h=0\}$ and with finite logarithmic stratification. If $f=h^{2} f_{0}$ is of finite relative codimension along $H$, then the restriction of $f_{0}$ to any logarithmic strata is a submersion except at the origin, that is, $\operatorname{dim}_{C}\left(\mathcal{O}_{x} /\left(\xi_{1} \cdot f_{0}, \ldots, \xi_{t} \cdot f_{0}\right)\right)<\infty$.

Proof. The conclusion means that for every $x_{0} \in H \backslash\{0\}$, there exists a vector field $\xi$ tangent to $H$ such that $\left(\xi \cdot f_{0}\right)\left(x_{0}\right) \neq 0$. Since $\operatorname{dim}_{C}\left(\mathcal{O}_{x} /\left(2 \alpha_{1} f_{0}+\right.\right.$ $\left.\left.\xi_{1} \cdot f_{0}, \ldots, 2 \alpha_{t} f_{0}+\xi_{t} \cdot f_{0}\right)\right)<\infty$ (by hypothesis), thus we obtain that $\operatorname{dim}_{C}\left(\mathcal{O}_{x} /\right.$ $\left.\left(f_{0}, \xi_{1} \cdot f_{0}, \ldots, \xi_{t} \cdot f_{0},\right)\right)<\infty$. If $\mathcal{O}_{x} /\left(\xi_{1} \cdot f_{0}, \ldots, \xi_{t} \cdot f_{0}\right)$ is not a finite dimensional vector space, then by the Curve Selection Lemma there is an analytic path $\Gamma$ on $H \backslash 0$ (since outside $H$ the $\xi_{i}$ 's form a generating family of the vector fields of the ambient space and $f_{0}$ has only isolated singularities), such that $0 \in \bar{\Gamma}$, which is contained in the support of the coherent $\mathcal{O}_{x}$-module sheaf induced from $\mathcal{O}_{x} /\left(\xi_{1} \cdot f_{0}, \ldots, \xi_{t} \cdot f_{0}\right)$. We can suppose that $\Gamma$ is contained in a single logarithmic stratum, say $\mathscr{S}$. Hence $d\left(f_{0_{\mid \mathscr{S}}}\right)$ vanishes along $\Gamma$. Since $f_{0}(0)=0$, $f_{0_{\mid \mathscr{S}}}=0$. But this is a contradiction to $\operatorname{dim}_{\boldsymbol{C}}\left(\mathcal{O}_{x} /\left(f_{0}, \xi_{1} \cdot f_{0}, \ldots, \xi_{t} \cdot f_{0}\right)\right)<\infty$.

Bruce and Roberts were just interested in the finite determinacy on the right on varieties. But it is easy to obtain from their work the notion of contact equivalence along a given variety for function germs having isolated singularities on the variety.

If we were interested in the contact finite determinacy of nonisolated hypersurface (that is $\operatorname{dim}_{C}\left(I^{2} / T_{e} \mathscr{K}_{I}(f)\right)<\infty$ ), then as a consequence of the above discussion and of the previous proof, we have

COROLlary 2.2. Let $f=h^{2} f_{0} \in I^{2}$. Then $f$ is of contact finite determinacy relative to $H$ if and only if $f_{0}$ is of finite contact determinacy along $H$. Moreover if the logarithmic stratification of $H=\{h=0\}$ is finite then $f_{0}$ is finitely determined on the right relative to $H$ if and only if $f_{0}$ is of finite contact determinacy along $H$, i.e.,

$$
\operatorname{dim}_{C} \frac{\mathcal{O}_{x}}{T_{e} \mathscr{R}_{I}\left(f_{0}\right)}<\infty \quad \text { if and only if } \operatorname{dim}_{C} \frac{\mathcal{O}_{x}}{T_{e} \mathscr{K}_{I}\left(f_{0}\right)}<\infty .
$$

\subsection{The smooth case}

In this section we deal with functions singular along a smooth hypersurface through the origin, i.e., $I:=\left(x_{n}\right)$. That is the first (and simplest) case to work 
with, and it really behaves as does any function with an isolated singularity along $H:=\left\{x_{n}=0\right\}$. The $\mathcal{O}_{x}$-module $\Theta_{I, e}$ is freely generated by $\partial / \partial x_{1}, \ldots, \partial / \partial x_{n-1}$ and $x_{n}\left(\partial / \partial x_{n}\right)$. We have a converse to Proposition 2.1.

Proposition 2.3. Let $f_{0}$ be a function with an isolated singularity at the origin along the hypersurface $\left\{x_{n}=0\right\}$. Then the function $f=x_{n}^{2} f_{0}$ is of finite relative codimension along $\left\{x_{n}=0\right\}$.

Proof. Let $\Gamma$ be the zeros set of $\partial f_{0} / \partial x_{1}, \ldots, \partial f_{0} / \partial x_{n-1}$. It is a onedimensional locus whose intersection with $H$ is reduced to the origin. We have to show that $\left\{2 f_{0}+x_{n}\left(\partial f_{0} / \partial x_{n}\right)=0\right\} \cap \Gamma$ is just the origin. Let $\Gamma=\Gamma_{1} \cup \cdots \cup$ $\Gamma_{k}$, where each $\Gamma_{i}$, for $i=1, \ldots, k$, is an irreducible curve germ at the origin with holomorphic parametrization $\gamma^{i}(z)$. We can suppose that $k=1$. By an easy calculus we find that

$$
\frac{d}{d z}\left[\left(x_{n}^{2} f_{0}\right) \circ \gamma\right](z)=\gamma_{n}(z) \frac{d \gamma_{n}}{d z}(z)\left[\left(2 f_{0}+x_{n} \frac{\partial f_{0}}{\partial x_{n}}\right) \circ \gamma(z)\right] .
$$

Since $\Gamma_{i}$ goes through $H$ only at the origin, if $z \neq 0$ then $\gamma_{n}(z)\left(d \gamma_{n} / d z\right)(z) \neq 0$. Thus

$$
\begin{aligned}
\left\{2 f_{0}+x_{n} \frac{\partial f_{0}}{\partial x_{n}}=0\right\} \cap \Gamma & =\left\{\frac{d}{d z}\left(\left(x_{n}^{2} f_{0}\right) \circ \gamma\right)=0\right\} \\
& =\left\{\left(x_{n}^{2} f_{0}\right) \circ \gamma=0\right\}=\left\{f_{0}(\gamma)=0\right\}
\end{aligned}
$$

which implies that $\left\{2 f_{0}+x_{n}\left(\partial f_{0} / \partial x_{n}\right)=0\right\} \cap \Gamma=\left\{\left(x_{n}\left(\partial f_{0} / \partial x_{n}\right)\right) \circ \gamma=0\right\}=\{0\}$ since $\partial f_{0} / \partial x_{n} \neq 0$ when all the other derivatives are vanishing outside $H$.

We have to remember that any $f_{0}$ which admits an isolated singularity along $\left\{x_{n}=0\right\}$ can be written as $g_{0}\left(x_{1}, \ldots, x_{n-1}\right)+x_{n} g_{1}(x)$, where $g_{0}$ admits an isolated singularity at the origin in $\left(\boldsymbol{C}^{n-1}, 0\right)$, and $g_{1}$ is such that there is a non-negative integer $k$ such that $\left(\partial_{1}^{k} g / \partial x_{n}^{k}\right)(0) \neq 0$, or there exists an $i \in\{1, \ldots, n-1\}$ such that $\left(\partial g_{1} / \partial x_{i}\right)(0) \neq 0$. These are necessary conditions on $g_{0}$ and $g_{1}$, but we do not think there are sufficient to produce a $f_{0}$ with an isolated singularity along $\left\{x_{n}=0\right\}$.

Proposition 2.4. With the above assumptions and notations, $R C_{F}$ is a smooth analytic set germ of dimension $p$ and thus $R D_{F}$ is a hypersurface.

Proof. We see that

$$
R C_{F}:=\left\{\frac{\partial F_{0}}{\partial x_{1}}=\cdots=\frac{\partial F_{0}}{\partial x_{n-1}}=2 F_{0}+x_{n} \frac{\partial F_{0}}{\partial x_{n}}=0\right\} .
$$

It is clear that $F_{0}$ is a versal deformation of the isolated hypersurface singularity $f_{0}^{-1}(0)$. So we can write $F_{0}(x, u)=f_{0}(x, u)+\sum_{k=1}^{p-1} u_{k} e_{k}(x)-u_{p}$. By [Tei, Section 5.5], we know that $\left\{\partial F_{0} / \partial x_{1}=\cdots=\partial F_{0} / \partial x_{n-1}=0\right\}$ is smooth. Then it 
is clear that $R C_{F}$ is also smooth, since $\left\{2 F_{0}+x_{n}\left(\partial F_{0} / \partial x_{n}\right)=0\right\}$ is a graph and tranverse to $\left\{\partial F_{0} / \partial x_{1}=\cdots=\partial F_{0} / \partial x_{n-1}=0\right\}$.

Remark 2.1. Note that $\mathcal{O}_{x, u} /\left(\partial F_{0} / \partial x_{1}, \ldots, \partial F_{0} / \partial x_{n-1}, 2 F_{0}+x_{n}\left(\partial F_{0} / \partial x_{n}\right)\right)$ is a reduced algebra. From now we denote it by $\mathcal{O}_{R C_{F}}$.

Let $\mathcal{O}_{R D_{F}}$ be defined by the 0 -th Fitting ideal of the $\mathcal{O}_{y, u}$-module $\tilde{F}_{*}\left(\mathcal{O}_{R C_{F}}\right)$. As usual, to know the structure of the discriminant with this structure sheaf, we need to know what the generic points of the discriminant are. Since there is only one logarithmic stratum contained in $H$ ( $H$ itself $),\left.f_{0}\right|_{H \backslash 0}$ is a submersion.

Let $\tilde{\mathcal{O}}$ be the sheaf of holomorphic function germs on $\boldsymbol{C}^{n} \times \boldsymbol{C}^{p}$.

Lemma 2.5. Let $f=x_{n}^{2} f_{0}$ of finite relative codimension along $\left\{x_{n}=0\right\}$. Let $F=x_{n}^{2} F_{0}$ be its mini-versal unfolding. Then there is a Zariski open dense subset of $R C_{F}$ of points $\left(x_{0}, u_{0}\right)=\left(x_{0}, u_{0}^{(1)}, \ldots, u_{0}^{(p)}\right)$ such that

$$
\operatorname{dim}_{C} \frac{\tilde{\mathcal{O}}_{\left(x_{0}, u_{0}\right)}}{\left(\partial f_{0} / \partial x_{1}, \ldots, \partial f_{0} / \partial x_{n-1}, 2 f_{0}+x_{n}\left(\partial f_{0} / \partial x_{n}\right), u_{1}-u_{0}^{(1)}, \ldots, u_{p}-u_{0}^{(p)}\right)}=1 .
$$

Proof. We find that

$$
R C_{F} \cap\left\{x_{n}=0\right\}=\left\{\frac{\partial F_{0}}{\partial x_{1}}=\cdots=\frac{\partial F_{0}}{\partial x_{n-1}}=F_{0}=x_{n}=0\right\} .
$$

Since $f_{0}=k_{0}\left(x_{1}, \ldots, x_{n-1}\right)+x_{n} k_{1}(x)$ such that $k_{0}$ admits an isolated singularity at the origin of $\left(\boldsymbol{C}^{n-1}, 0\right)$, then we can write

$$
F_{0}(x, u)=K_{0}\left(x_{1}, \ldots, x_{n-1}, u\right)+x_{n} K_{1}(x, u) .
$$

It is obvious that $K_{0}$ is a $\mathscr{K}_{e}$-versal unfolding of the isolated hypersurface singularity $k_{0}^{-1}(0)$. Thus

$$
R C_{F} \cap\left\{x_{n}=0\right\}=\left\{\frac{\partial K_{0}}{\partial x_{1}}=\cdots=\frac{\partial K_{0}}{\partial x_{n-1}}=K_{0}=x_{n}=0\right\} .
$$

This is a smooth germ in $\left(\boldsymbol{C}^{n-1} \times 0 \times \boldsymbol{C}^{p}, 0\right)$ of dimension $p-1$. This exactly means that

$$
R C_{F} \cap\left\{x_{n} \neq 0\right\}=\left\{\frac{\partial F}{\partial x_{1}}=\cdots=\frac{\partial F}{\partial x_{n}}=0: x_{n} \neq 0\right\} .
$$

Then the set outside $\left\{x_{n} \neq 0\right\}$ where the $f_{u}$ 's have only Morse singular points with $p$ distinct critical values is a Zariski dense open subset of $R C_{F} \cap\left\{x_{n} \neq 0\right\}$. Since $R C_{F}$ is irreducible and $\operatorname{dim} R C_{F} \cap\left\{x_{n}=0\right\} \leq p-1$, there is a Zariski dense open subset of $R C_{F}$ where the $f_{u}$ 's have only Morse singular points with $p$ distinct critical values, which ends the proof.

So, for a function germ $f$ of finite relative codimension along $H$ equal to 1 , we obtain that the maximal ideal of $\mathcal{O}_{x}, m_{x}=\left(\partial f_{0} / \partial x_{1}, \ldots, \partial f_{0} / \partial x_{n-1}, 2 f_{0}+\right.$ 
$\left.x_{n}\left(\partial f_{0} / \partial x_{n}\right)\right)$. If $f_{0} \in m_{x}^{2}$, then since $2 f_{0}+x_{n}\left(\partial f_{0} / \partial x_{n}\right) \in m_{x}^{2}$, we obtain that $m_{x} \subset$ $\left(\partial f_{0} / \partial x_{1}, \ldots, \partial f_{0} / \partial x_{n-1}\right)+m_{x}^{2}$ and so by Nakayama's Lemma $m_{x}=\left(\partial f_{0} / \partial x_{1}, \ldots\right.$, $\left.\partial f_{0} / \partial x_{n-1}\right)$ which is impossible. Since the previous dimension is positive, we deduce that $f_{0} \in m_{x} \backslash m_{x}^{2}$ with $\left(\partial f_{0} / \partial x_{n}\right)(0) \neq 0$. In expanding $f_{0}$ as a power series in $x_{n}$ and in making explicit the equations of $\left(\partial f_{0} / \partial x_{1}, \ldots, \partial f_{0} / \partial x_{n-1}, 2 f_{0}+\right.$ $\left.x_{n}\left(\partial f_{0} / \partial x_{n}\right)\right)=m_{x}$, we find that $f_{0}(x)=k_{0}\left(x_{1}, \ldots, x_{n-1}\right)+x_{n} k_{1}(x)$ where $k_{0}$ is a Morse function in the variables $x_{1}, \ldots, x_{n-1}$, and $k_{1}$ is invertible.

Proposition 2.6. The residual discriminant $R D_{F}$ endowed with the ringed structure $\mathcal{O}_{R D_{F}}=\mathcal{O}_{y, u} / \tilde{F}_{0}\left(\tilde{F}_{*} \mathcal{O}_{R C_{F}}\right)$ is irreducible and reduced.

Proof. The irreducibility comes from the smoothness of $R C_{F}$. To show that $\mathcal{O}_{R D_{F}}$ is reduced, there is just to see that it is reduced at a smooth point, see [dPGW, Corollary 1.18]. Let $f(x)=x_{n}^{2}\left(x_{n}+x_{1}^{2}+\cdots+x_{n-1}^{2}\right)$ be a generic point of the residual discriminant. Then $F(x, u)=x_{n}^{2}\left(x_{n}+x_{1}^{2}+\cdots+x_{n-1}^{2}-u\right)$ is a mini-versal unfolding of $f$. We see that $\mathcal{O}_{R C_{F}}=\mathcal{O}_{x, u} /\left(x_{1}, \ldots, x_{n-1}, 3 x_{n}-2 u\right)$, and by computations we have $\mathscr{F}_{0}\left(\tilde{F}_{*} \mathcal{O}_{R C_{F}}\right)$ is generated by $y-(4 / 27) u^{3}$, hence reduced.

Another important question about the geometry of the (residual) discriminant is to exhibit a basis of the vector fields tangent to the (residual) discriminant in order to know if it is a free divisor, and if such vector fields are liftable by the unfolding map associated to the mini-versal unfolding, since this is the case for isolated complete intersection singularities (see [Lo, Corollary 6.13] and [Go, Theorem 3] or [Sa1], [Ter1], [Bru1] for hypersurfaces). As in the isolated singualirity case, instead of considering the mini-versal unfolding we consider the truncated mini-versal unfolding:

Definition 2.7. Let $f=h^{2} f_{0}$ be a function of finite relative codimension along $\left\{x_{n}=0\right\}$ equal to $p$. Let $h^{2} e_{1}, \ldots, h^{2} e_{p}$ be a $C$-basis of $I^{2} / T_{e} \mathscr{R}_{I}(f)$, with $e_{p}(x)=-1$. Then the unfolding $G(x, v)=h^{2}(x)\left[f_{0}(x)+\sum_{i=1}^{p-1} v_{i} e_{i}(x)\right]$ is called the truncated mini-versal unfolding of $f$.

Now we return to the case $h(x)=x_{n}$. To make the distinction between the truncated mini-versal unfolding $G$ and the mini-versal unfolding $F$ of $f$, we will denotes $R C_{G}, R C_{F}, R D_{G}$ and $R D_{F}$ their respective residual critical loci and discriminants.

Then $R C_{G}$ is the support of the coherent $\mathcal{O}_{x, v}$-module sheaf generated by

$$
M_{R C_{G}}:=\frac{I^{2} \otimes_{\mathcal{O}_{x}} \mathcal{O}_{x, v}}{\left\{Y \cdot G: Y \in \Theta_{H} \otimes_{\mathcal{O}_{x}} \mathcal{O}_{x, v}\right\}}
$$

and $R D_{G}$ is the image of $R C_{G}$ by $\tilde{G}:=G \times i d_{\left(C^{p-1}, 0\right)}$.

Let $F(x, u)=x_{n}^{2}\left[f_{0}(x)+\sum_{i=1}^{p-1} u_{i} e_{i}(x)+u_{p} e_{p}(x)\right]=x_{n}^{2} F_{0}(x)$. Such an $F_{0}$ is a versal unfolding of the isolated hypersurface singularity $f_{0}^{-1}(0)$. Let us define 
the function $G_{0}(x, v)$ such that $G_{0}(x, v)+u_{p} e_{p}(x):=F_{0}(x, u)$. Then $G(x, v)=$ $x_{n}^{2} G_{0}(x, v)$. By easy calculus, we obtain

$$
R C_{G}=\left\{(x, v): \frac{\partial G_{0}}{\partial x_{1}}=\cdots=\frac{\partial G_{0}}{\partial x_{n-1}}=2 G_{0}+x_{n} \frac{\partial G_{0}}{\partial x_{n}}=0\right\} .
$$

We define $\mathcal{O}_{R C_{G}}:=\mathcal{O}_{x, v}\left(\partial G_{0} / \partial x_{1}, \ldots, \partial G_{0} / \partial x_{n-1}, 2 G_{0}+x_{n}\left(\partial G_{0} / \partial x_{n}\right)\right)$ and $\mathcal{O}_{R D_{G}}:=$ $\mathcal{O}_{x, v} / \mathscr{F}_{0}\left(\tilde{G}_{*} \mathcal{O}_{R C_{G}}\right)$. We have to note that $\mathcal{O}_{R C_{G}}$ defines a complete intersection. The first thing to see is that the codimension of $R C_{G}$ is $\leq n$. Since

$$
R C_{G} \cap\left\{v_{1}=\cdots=v_{p-1}=0\right\}=\operatorname{supp} \frac{m_{x} I^{2}}{T_{e} \mathscr{R}_{I}(f)}=\{0\}
$$

we deduce from this that the dimension of $R C_{G}$ is $\leq p-1$, and so $R C_{G}$ becomes a (non-reduced) complete intersection of dimension $p-1$. The consequence of this is that $M_{R C_{G}}$ is a free $\mathcal{O}_{v}$-module of rank $p-1$. This also prove that we can define $\mathcal{O}_{R D_{G}}$ as done above.

We would like to know more about how to obtain some gemetrically important sets for $F$ from those corresponding to $G$.

Let $\chi$ be the vector field on $\left(\boldsymbol{C} \times \boldsymbol{C}^{p}, 0\right)$ such that $\chi \circ \tilde{F}(x, u)=-x_{n}^{2}(\partial / \partial y)+$ $\left(\partial / \partial u_{p}\right)=d \tilde{F} \cdot\left(\partial / \partial u_{p}\right)$. Let us denote by $\Psi(y, u, t)$ the local flow of $\chi$, with the initial condition $\Psi(y, u, 0)=\left(y, v, 2 u_{p}\right)$, then $\Psi(y, u, t)=\left(\Psi_{0}(y, u, t), v, t+2 u_{p}\right)$ and

$$
\Psi(\tilde{F}(x, u), u, t)=\left(F(x, u)-t x_{n}^{2}, v, t+2 u_{p}\right) .
$$

Let $\Phi(y, u):=\Psi\left(y, u,-u_{p}\right)=\left(\Psi_{0}\left(y, u,-u_{p}\right), u\right)=\left(\Phi_{0}(y, u), u\right)$. Then we have $\operatorname{Im}(\tilde{G}) \times \boldsymbol{C}=\Phi(\operatorname{Im}(\tilde{F}))$ and $\Phi$ is a well-defined diffeomorphism of $\boldsymbol{C} \times \boldsymbol{C}^{p}$ preserving the origin. Let $C_{F}$ be the projection of $R C_{F}$ on $\left(\boldsymbol{C}^{n} \times \boldsymbol{C}^{p-1}, 0\right)$, in forgetting the last coordinate $u_{p}$. Then we have

$$
\Phi\left(\tilde{F}\left(R C_{F}\right)\right)=\Phi\left(R D_{F}\right)=\tilde{G}\left(C_{F}\right) \times C .
$$

Since $R D_{G} \times\{0\}=R D_{F} \cap\left\{u_{p}=0\right\}$ and $\Phi(y, v, 0)=(y, v, 0)$, from this, we deduce that

$$
R D_{G} \times\{0\}=R D_{F} \cap\left\{u_{p}=0\right\}=\Phi\left(R D_{F} \cap\left\{u_{p}=0\right\}\right)=\tilde{G}\left(C_{F}\right) \times\{0\} .
$$

This is the fundamental point to know whether or not $R D_{F}$ is a free divisor. Now we state

THEOREM 2.8. $R D_{G}$ is a free divisor.

The next theorem follows from the proof of Theorem 2.8.

THEOREM 2.9. $R D_{F}$ is a free divisor.

Proof of Theorem 2.8. The method to produce a generating family of vector fields tangent to the discriminant of a projection of an isolated complete intersection singularity is now usual (see also [Bru1], [Ter2] and [Go]). Note that 
$M_{R C_{G}}$ is an $\mathcal{O}_{v}$-free module of rank $p-1$. Then for each $j=1, \ldots, p$, we have

$$
G x_{n}^{2} e_{j}=\sum_{i=1}^{p} a_{i, j}(v) x_{n}^{2} e_{i} \text { in } M_{R C_{G}}
$$

which gives

$$
\sum_{i=1}^{p}\left[a_{i, j}(v)-G(x, v) \delta_{i, j}\right] x_{n}^{2} e_{i}+\sum_{k=1}^{n-1} b_{k, j}(x, v) \frac{\partial G}{\partial x_{k}}+b_{j}(x, v) x_{n} \frac{\partial G}{\partial x_{n}}=0 .
$$

If $A_{G}$ is the $p \times p$-matrix with entries the $\left(a_{i, j}(v)-y \delta_{i, j}\right)$ 's, then $\operatorname{det} A_{G}=0$ is an equation of the residual discriminant according to Theorem 1.4. Let $\chi_{i} \in \Theta_{x, v}$ be the following vector field

$$
\chi_{j}(x, v)=\sum_{i=1}^{p-1}\left[a_{i, j}(v)-G(x, v) \delta_{i, j}\right] \frac{\partial}{\partial v_{i}}+\sum_{k=1}^{n-1} b_{k, j}(x, v) \frac{\partial}{\partial x_{k}}+b_{j}(x, v) x_{n} \frac{\partial}{\partial x_{n}} .
$$

Thus we have $\chi_{j} \cdot G=-\left(a_{j, p}-G \delta_{j, p}\right)$ and $\chi_{j} \cdot u_{l}=\sum_{i=1}^{p-1}\left[a_{i, j}(v)-G(x, v) \delta_{i, j}\right] \delta_{i, l}=$ $\left(a_{l, j}-G \delta_{l, j}\right)$. Let $\eta_{i}$ be the vector field of $\Theta_{y, v}$ defined by

$$
\eta_{j}(y, v)=-\left(a_{p, j}(v)-y \delta_{p, j}\right) \frac{\partial}{\partial y}+\sum_{i=1}^{p-1}\left[a_{i, j}(v)-y \delta_{i, j}\right] \frac{\partial}{\partial v_{i}} .
$$

Then the $\eta_{i}$ 's are tangent to $R D_{F}(G)$ and $d \tilde{G}_{\chi_{i}}=\eta_{i} \circ \tilde{G}$. To conclude it is sufficient to show that

Lemma 2.10. The $\eta_{i}$ 's are the generators of the $\mathcal{O}_{y, v}$-module $\Theta_{R D_{G}}$.

Proof. The proof is inspired by that of [Go, Theorem 3.1]. First we have to note that outside $R D_{G}$ the $\eta_{i}$ 's are linearly independant. Let $\eta$ be any vector field of $\Theta_{y, v}$ tangent to the residual discriminant $R D_{G}$. We form the $p \times(p+1)$-matrix whose $p$ first columns are the coefficients of the vector fields $\eta_{i}$ 's and the last one is given by the coefficients of $\eta$. To finish the proof of this lemma we need the following

Lemma 2.11. $\mathcal{O}_{R D_{G}}$ is reduced.

Proof. Since $R D_{G} \times C$ is isomorphic to $R D_{F}$ by $\Phi$ then we have the following isomorhism of local $\boldsymbol{C}$-algebras

$$
\Phi^{*}: \mathcal{O}_{R D_{F}} \rightarrow \mathcal{O}_{R D_{G} \times C}=\mathcal{O}_{R D_{G}} \otimes_{\mathcal{O}_{y, v}} \mathcal{O}_{y, u} .
$$

Let $k \in \mathcal{O}_{R D_{G}}$ such that $k \neq 0$ and $k^{d}=0$ for a positive integer $d$. This implies by the $\left(\Phi^{*}\right)^{-1}\left(k^{d}\right)=0$ and so $\left(\Phi^{*}\right)^{-1}(k)=0$, which ends the proof.

End of proof of Lemma 2.10. By Lemma 2.11 and Theorem 1.4, $\left\{\operatorname{det} A_{G}=0\right\}$ gives a reduced equation of $R D_{G}$. Each $p \times p$ minor containing 
the last column is of the form $\phi_{j}(y, v) \Delta(y, v)$, where $\{\Delta(y, v)=0\}$ is a reduced equation of $R D_{G}$. Thus the vector field $\eta-\sum_{j=1}^{p}(-1)^{p-j} \phi_{j} \eta_{j}$ vanishes outside $R D_{G}$, and since the $\eta_{i}$ 's are a free basis of the vector fields in $\boldsymbol{C} \times \boldsymbol{C}^{p-1} \backslash R D_{G}$, by continuity $\eta=\sum_{j=1}^{p}(-1)^{p-j} \phi_{j} \eta_{j}$.

To finish this subsection we give a basis of the vector fields tangent to $R D_{F}$. For any $j=1, \ldots, p$, we have

$$
F x_{n}^{2} e_{j}=\sum_{i=1}^{p} \alpha_{i, j}(u) x_{n}^{2} e_{i}+\sum_{k=1}^{n-1} \beta_{k, j}(x, u) \frac{\partial F}{\partial x_{k}}+\beta_{j}(x, u) x_{n} \frac{\partial F}{\partial x_{n}}
$$

and thus

$$
0=\sum_{i=1}^{p}\left[\alpha_{i, j}(u)-F(x, u) \delta_{i, j}\right] x_{n}^{2} e_{i}+\sum_{k=1}^{n-1} \beta_{k, j}(x, u) \frac{\partial F}{\partial x_{k}}+\beta_{j}(x, u) x_{n} \frac{\partial F}{\partial x_{n}} .
$$

Now we define the vector fields $\Lambda_{i}$ in $\left(\boldsymbol{C}^{n+p}, 0\right)$ and $\Gamma_{i}$ in $\left(\boldsymbol{C} \times \boldsymbol{C}^{p}, 0\right)$ for $i=1, \ldots, p$, by

$$
\begin{aligned}
& \Lambda_{j}=\sum_{i=1}^{p-1}\left[\alpha_{i, j}(u)-F(x, u) \delta_{i, j}\right] \frac{\partial}{\partial u_{i}}+\sum_{k=1}^{n-1} \beta_{k, j}(x, u) \frac{\partial}{\partial x_{k}}+\beta_{j}(x, u) x_{n} \frac{\partial}{\partial x_{n}}, \\
& \Gamma_{j}=-\left(\alpha_{p, j}(u)-y \delta_{p, j}\right) \frac{\partial}{\partial y}+\sum_{i=1}^{p-1}\left[\alpha_{i, j}(u)-y \delta_{i, j}\right] \frac{\partial}{\partial u_{i}} .
\end{aligned}
$$

We have $d \tilde{F} \cdot \Lambda_{i}=\Gamma_{i} \circ \tilde{F}$. When the $\chi_{i}$ 's, vector fields in $\left(\boldsymbol{C}^{n} \times \boldsymbol{C}^{p-1}, 0\right)$, are seen as vector fields in $\left(\boldsymbol{C}^{n} \times \boldsymbol{C}^{p-1} \times \boldsymbol{C}, 0\right)$, we also have $\Lambda_{i}(x, v, 0)=\chi_{i}(x, v)$ and that $\Gamma_{i} \circ(G(x, v), v, 0)=\eta_{i} \circ(G(x, v), v)$, if we also consider the $\eta_{i}$ 's as vector fields in $\left(\boldsymbol{C} \times \boldsymbol{C}^{p-1} \times \boldsymbol{C}, 0\right)$. Now to finish to find a basis of the $\mathcal{O}_{y, u}$-module of the vector field tangent to $R D_{F}$ we need to know the Jacobian matrix of $\Phi$ denoted by $A$ and its inverse $A^{-1}$

$$
\begin{aligned}
A & =\left[\begin{array}{ccccc}
\frac{\partial \Phi_{0}}{\partial y} & \frac{\partial \Phi_{0}}{\partial u_{1}} & \frac{\partial \Phi_{0}}{\partial u_{2}} & \cdots & \frac{\partial \Phi_{0}}{\partial u_{p}} \\
0 & 1 & 0 & \ldots & 0 \\
\vdots & \ddots & \ddots & \ddots & \vdots \\
\vdots & & \ddots & 1 & 0 \\
0 & \ldots & \cdots & 0 & 1
\end{array}\right] \\
A^{-1} & =\frac{1}{\partial \psi_{0} / \partial y}\left[\begin{array}{ccccc}
\gamma_{0} & \gamma_{1} & \gamma_{2} & \cdots & \gamma_{p} \\
0 & 1 & 0 & \cdots & 0 \\
\vdots & \ddots & \ddots & \ddots & \vdots \\
\vdots & & \ddots & 1 & 0 \\
0 & \ldots & \ldots & 0 & 1
\end{array}\right]
\end{aligned}
$$


where $\gamma_{0}=\left(\partial \Phi_{0} / \partial y\right)^{-1} \quad$ and $\quad \gamma_{i}=-\gamma_{0}\left(\partial \Phi_{0} / \partial u_{i}\right) \quad$ for $\quad 1 \leq i \leq p$. From $\Phi_{0}(F(x, u), u)=G(x, v)$, we deduce

$$
\begin{aligned}
\frac{\partial \Phi_{0}}{\partial u_{p}}(F(x, u), u) & =x_{n}^{2} \frac{\partial \Phi_{0}}{\partial y}\left(F(x, u), u,-u_{p}\right) \text { and so we obtain } \\
d\left(\Phi^{-1}\right) \frac{\partial}{\partial u_{p}}(y, u) & =\left(\frac{\partial \Phi_{0}}{\partial y}\right)^{-1}(y, u)\left[-\frac{\partial \Phi_{0}}{\partial u_{p}}(y, u) \frac{\partial}{\partial y}+\frac{\partial}{\partial u_{p}}\right] \\
d\left(\Phi^{-1}\right) \frac{\partial}{\partial u_{p}}(G(x, v), u) & =\left[\frac{1}{\partial \Phi_{0} / \partial y} \chi\right] \circ(F(x, u), u) .
\end{aligned}
$$

Since $\chi$ is tangent to $R D_{F}$ by the above computations and since the determinant of the matrix formed by the $\Gamma_{i}$ 's and $\chi$ is a reduced equation of the free divisor $R D_{F}$, then by Saito's Lemma [Sa1, Lemma 1.9], we have proved the following

Proposition 2.12. The vector fields $\Gamma_{1}, \ldots, \Gamma_{p}$ and $\chi$ of $\Theta_{y, u}$ generate freely the $\mathcal{O}_{y, u}$-module of the vector fields tangent to $R D_{F}$, and are liftable by $\tilde{F}$.

\subsection{The free divisor case}

We recall that a free divisor is a hypersurface whose module of tangent vector fields $\left(\Theta_{I, e}\right)$ is a free $\mathcal{O}_{x}$-module.

We say that a hypersurface $H$ (or a principal ideal $I$ ) is Euler if there is a vector field $E$ tangent to $H$ such that $E \cdot I=I$. This means that we can choose such a vector field such that, given a generator $h$ of $I$, then $E \cdot h=h$; we denote this vector field by $E_{h}$. In that case $\Theta_{I, e}=\Theta_{h}^{0} \oplus \mathcal{O}_{x} E_{h}$, where $\Theta_{h}^{0}:=$ $\{\xi: \xi \cdot h=0\}$, see $[\mathrm{All}$, p. 2] or [DM, Lemma 3.3]. Then an Euler hypersurface is a free divisor if and only if $\Theta_{h}^{0}$ is an $\mathcal{O}_{x}$-free module.

Now we suppose that $H$ is a free divisor and Euler. Let $h$ be a given reduced equation of $H$. Let $\xi_{1}, \ldots, \xi_{n-1}$ be a system of generators of $\Theta_{h}^{0}$. The next proposition is a kind of converse to Proposition 2.1. We need some notations to state it.

Let $f_{0} \in \mathcal{O}_{x}$ with $\operatorname{dim}_{C}\left(\mathcal{O}_{x} /\left(\xi_{1} \cdot f_{0}, \ldots, \xi_{n-1} \cdot f_{0}, E_{h} \cdot f_{0}\right)\right)$ finite. Let us denote by $\Gamma$ the 1 -dimensional complete intersection $\left\{\xi_{1} \cdot f_{0}=\cdots=\xi_{n-1} \cdot f_{0}=0\right\}$. Then $\Gamma$ is a finite union of irreducible analytic curves $\Gamma_{1}, \ldots, \Gamma_{s}$. For $i=1, \ldots, s$, let $\gamma^{(i)}$ be a complex analytic parametrization of the curve $\Gamma_{i}$.

Proposition 2.13. Let $H$ be a free divisor and Euler with reduced equation $h$. Let $f_{0} \in \mathcal{O}_{x}$ with $\operatorname{dim}_{C}\left(\mathcal{O}_{x} /\left(\xi_{1} \cdot f_{0}, \ldots, \xi_{n-1} \cdot f_{0}, E_{h} \cdot f_{0}\right)\right)$ finite. Let us consider the following conditions:

(i) $\xi_{1} \cdot f_{0}, \ldots, \xi_{n-1} \cdot f_{0}, h$ is a regular sequence,

(ii) $\Gamma \cap H=\Gamma_{1} \cup \ldots \cup \Gamma_{t}$ and for each $i=1, \ldots, t$

$$
\frac{d \gamma^{(i)}}{d z}(z) \in \operatorname{span}\left(\xi_{1} \circ \gamma^{(i)}(z), \ldots, \xi_{n-1} \circ \gamma^{(i)}(z), E_{h} \circ \gamma(z)\right),
$$

(iii) There exist a finite number of logarithmic strata of $H$ which meet $\Gamma$. 
If $f_{0}$ satisfies one of the above conditions, then $f:=h^{2} f_{0}$ is of finite relative codimension along $H$.

Proof. (i) $\Gamma$ is one-dimensional and we can suppose it is irreducible and parametrized by the analytic arc germ $\gamma$, with $\gamma(0)=0$. By hypothesis $\Gamma \cap H=$ $\{0\}$. Then $h(\gamma(z))=0$ if and only if $z=0$.

Let $M(x)$ be the $n \times n$-matrix whose rows are the coefficients of the vector fields $\xi_{1}, \ldots, \xi_{n-1}$ and $\xi_{n}:=E_{h}$. Let us denote by $m_{i, j}$ the $(n-1) \times(n-1)$ minor obtained from $M$ by deleting the $i$-th row and the $j$-th column. Remember that det $M(x)=u(x) h(x)$ where $u$ is a unit. Along $\Gamma \backslash\{0\}$, the vector fields $\xi_{1}, \ldots, \xi_{n-1}$ and $E_{h}$ are linearly independent. Thus for any $k=1, \ldots, n$ we have

$$
u(\gamma(z)) h(\gamma(z)) \frac{\partial}{\partial x_{k}} \circ \gamma(z)=\sum_{l=1}^{n}(-1)^{l+k} m_{l, k}(\gamma(z)) \xi_{l} \circ \gamma(z) .
$$

Now we have

$$
\frac{d}{d z}\left(\left(h^{2} f_{0}\right) \circ \gamma(z)\right)=h \circ \gamma(z)\left[2 f_{0} d_{\gamma(z)} h \cdot \gamma^{\prime}(z)+h(\gamma(z)) d_{\gamma(z)} f_{0} \cdot \gamma^{\prime}(z)\right],
$$

and using the above expression of the $\left(\partial / \partial x_{k}\right)$ 's we find

$$
\frac{d}{d z}\left(\left(h^{2} f_{0}\right) \circ \gamma\right)=\left[\frac{h}{u} \circ \gamma(z)\right]\left[\sum_{k=1}^{n}(-1)^{k+n} m_{k, n}(\gamma(z)) \gamma_{k}^{\prime}(z)\right]\left[\left(2 f_{0}+E_{h} \cdot f_{0}\right) \circ \gamma(z)\right] .
$$

We see that $\left[\sum_{k=1}^{n}(-1)^{k+n} m_{n, k}(\gamma(z)) \gamma_{k}^{\prime}(z)\right]$ is the determinant of the $n \times n$-matrix whose $(n-1)$-th first rows are the coefficients of $\xi_{1}, \ldots, \xi_{n-1}$ and the last one is made with the coefficients of $\gamma^{\prime}(z)$. Since the vector fields $\xi_{1}, \ldots, \xi_{n-1}$ are a basis of the vector fields tangent to the levels of $h$ and since $\Gamma \cap H=\{0\}$ the curve $\gamma$ is transverse to the levels $\left\{h=w_{0}(\neq 0)\right\}$ when $w_{0}$ is ranging a small open punctured neighbourhood of the origin in $\boldsymbol{C}$. Thus this determinant is non-zero. Thus $\left(2 f_{0}+E_{h} \cdot f_{0}\right) \circ \gamma(z)=0$ if and only if $(\partial / \partial z)\left(\left(h^{2} f_{0}\right) \circ \gamma(z)\right)=0$ if and only if $f_{0} \circ \gamma(z)=0$. Then $\left(2 f_{0}+E_{h} \cdot f_{0}\right) \circ \gamma(z)=0$ if and only if $\left(E_{h} \cdot f_{0}\right) \circ \gamma(z)=0$, which is by hypothesis $z=0$.

(ii) Let us suppose that $t=1$ and $\left(2 f_{0}+E_{h} \cdot f_{0}\right) \circ \gamma(z) \equiv 0$, with $\gamma^{(1)}=\gamma$. By hypothesis there are analytic functions in $z \alpha_{1}, \ldots, \alpha_{n-1}, \alpha$ such that

$$
\frac{d\left(f_{0} \circ \gamma\right)}{d z}(z)=\sum_{k=1}^{n-1} \alpha_{k}(z)\left(\xi_{k} \cdot f_{0}\right) \circ \gamma(z)+\alpha(z)\left(E_{h} \cdot f_{0}\right) \circ \gamma(z) .
$$

Thus we obtain the following differental equation $2 \alpha(z) f_{0} \circ \gamma(z)+\left(d\left(f_{0} \circ \gamma\right) /\right.$ $d z)(z)=0$. Since $f_{0} \circ \gamma(0)=0$, this implies that $f_{0} \circ \gamma \equiv 0$ and so $E_{h} \cdot f_{0} \circ \gamma \equiv 0$, which a contradiction to the hypothesis we have on $f_{0}$.

(iii) We can suppose that there is only one logarithmic stratum which meets $\Gamma$ and so we are in the case (ii). 
We have the following

Proposition 2.14. When $H$ is a free divisor, then residual Jacobian module $M_{R C_{F}}$ is a Cohen-Macaulay $\mathcal{O}_{x, u}$-module of dimension $p$.

Proof. Since $\Theta_{I, e}$ is free as $\mathcal{O}_{x}$-module (then $\left.t=n\right), M_{R C_{F}}$ is an $\mathcal{O}_{x, u}$-module canonically isomorphic to $\mathcal{O}_{x, u} /\left(A_{1}, \ldots, A_{n}\right)$. Since $R C_{F}$ is of dimension $\leq p$, $\mathcal{O}_{x, u} /\left(A_{1}, \ldots, A_{n}\right)$ is necessarily of dimension $p$. This means that $A_{1}, \ldots, A_{n}$ is an $\mathcal{O}_{x, u}$-regular sequence and then the depth and dimension of $\mathcal{O}_{x, u} /\left(A_{1}, \ldots, A_{n}\right)$ as $\mathcal{O}_{x, u}$-module are equal to $p$.

As it was done in the smooth case, given a mini-versal unfolding $F$ of a finite relative codimensional germ $f$ along $H$, we introduce the truncated mini-versal unfolding $G$ and then we define, as in the smooth case, the $\mathcal{O}_{x, v}$-module $M_{R C_{G}}$, the analytic sets $R C_{G}$ and $R D_{G}$ and the local analytic algebras $\mathcal{O}_{R C_{G}}$ and $\mathcal{O}_{R D_{G}}$. As in the smooth case we prove that $R D_{F} \cong R D_{G} \times C$ by an automorphism of $\mathcal{O}_{y, u}$ built by means of the vector field $\chi$ defined as $\chi \circ \tilde{F}=\mathrm{d} \tilde{F}\left(\partial / \partial u_{p}\right)$.

Damon in his trilogy [Da-I], [Da-II], [Da-III] computed discriminant and bifurcation locus of a versal unfolding of a mapping $\boldsymbol{C}^{k} \rightarrow \boldsymbol{C}^{l}$ under various equivalences preserving a variety (a free divisor or a free complete intersection) at the source or at the target. He noticed that there are such varieties on which there is no mapping whose discriminant is a free divisor. The explanation of this is that the variety has to present what he called generic Morse-type singularities, which is equivalent (when some conditions on $k$ or $l$ and numbers related to the geometry of the logarithmic stratification of the variety are satisfied) to the existence of functions of (extended) codimension one for the equivalences he considered. This condition is sufficient to provide a reduced equation to the discriminant by means of Fitting ideals, which here means the freeness. In our context the same kind of phenomenon appears. Let us consider the following

Example I. Let $h(x, y)=x^{3}+y^{3}$. It defines an Euler free divisor in $\boldsymbol{C}^{2}$. The tangent vector fields are generated by

$$
E=\frac{1}{3}\left(x \frac{\partial}{\partial x}+y \frac{\partial}{\partial y}\right) \text { and } \xi=y^{2} \frac{\partial}{\partial x}-x^{2} \frac{\partial}{\partial y} .
$$

Let $f_{0}(x, y)=x$ and $f(x, y)=h^{2} f_{0}$. Then, $f$ is of codimension 2 along the Euler free divisor $H:=\{h=0\} . \quad T_{e} \mathscr{R}_{I}(f)$ is the ideal generated by $x h^{2}$ and $y^{2} h^{2}$. Let $F_{0}(x, y, u)=f_{0}(x, y)+u_{1} y+u_{2}$. Then $F=h^{2} F_{0}$ is a mini-versal unfolding of $f$. Let $G$ be the truncated mini-versal unfolding of $f$. We find that $R C_{G}:=\{x=$ $y=0\} \subset C^{3}$. So $R D_{G}$ is just the $u_{1}$-axis in $C^{2}$. When we compute a resolution of $M_{R C_{G}}$ as an $\mathcal{O}_{t, u_{1}}$-module, where $T$ is coordinate at the origin of the target space $(\boldsymbol{C}, 0)$. Then the matrix whose determinant is the 0 -th Fitting ideal of the resolution is $t^{2} \mathrm{Id}$ (this is the matrix of the $\mathcal{O}_{u_{1}}$-endomorphism of $M_{R C_{G}}$ of multiplication by $G) . R D_{G}$ is a free divisor, but the Fitting ideal does not provide a reduced ideal. Note that $R C_{G} \subset H \times C$. 
Example II. This is true for any Euler free divisor $x^{k}+y^{l}$, with $k>l>2$ and $f(x, y)=x$. The smallest relative codimension along $H$ is $l-1$. The truncated residual discriminant is always the hyperplane of the truncated parameter space. But the 0 -th Fitting ideal is generated by $t^{l-1}$, not reduced. In that case $R C_{G} \subset H \times C$.

Example III. As above it is easy to see that when the Euler free divisor is of the form $x^{2}+y^{k}, k>1$, then this time everything works as in the smooth case.

The following proposition is a first step to understand what could be the relevant phenomena seen above.

Proposition 2.15. Let $H$ be an Euler free divisor. Let $f$ be a function of finite relative codimension $p$ along $H$ and let $G$ be a truncated mini-versal unfolding. Then the following sequence of $\mathcal{O}_{y, v}$-modules is exact:

$$
0 \rightarrow \mathscr{L} \rightarrow \mathcal{O}_{y, v}^{p} \stackrel{\alpha}{\rightarrow} M_{R C_{G}} \rightarrow 0 .
$$

Then

(i) $\mathscr{L}$ is the free $\mathcal{O}_{y, v}$-module of rank $p$ of the vector fields tangent to $R D_{G}$ which are liftable along $H \times C^{p-1}$. Moreover $\mathscr{L}$ is a Lie algebra.

(ii) Let $F$ be a mini-versal unfolding giving the previous $G$. If the dimension of $R C_{F} \cap\left(H \times C^{p}\right)$ is $\leq p-1$, then any vector field tangent to $R D_{F}$ is liftable by $\tilde{F}$.

Proof. (i) The freeness of $\mathscr{L}$ comes from the fact that $M_{R C_{G}}$ is CohenMacaulay of dimension $p-1$ and is a free $\mathcal{O}_{v}$-module of rank $p$. If we denote by $v_{p}$ the coordinate $y$, the map $\alpha$ sends $\partial / \partial v_{i}$ on $e_{i}$. For a vector field $\zeta \in \Theta_{y, v}$, $\alpha(\zeta)=0$ if and only if there exists a vector field $\eta \in \Theta_{x, v}$ tangent to $H \times C^{p-1}$ such that $\mathrm{d} \tilde{G} \eta=\zeta \circ \tilde{G}$. Note that such an $\mathscr{L}$ is a Lie algebra because of the last equality, which goes through the Lie bracket (see [Da-I, 3.3 (ii)]).

(ii) By hypothesis any vector field defined on $\boldsymbol{C} \times \boldsymbol{C}^{p} \backslash \tilde{F}\left(R C_{F} \cap\left(H \times \boldsymbol{C}^{p}\right)\right)$ can be uniquely extended to a vector field in $\Theta_{y, u}$ by the Hartogs Theorem since $\Delta:=\tilde{F}\left(R C_{F} \cap\left(H \times C^{p}\right)\right)$ is of codimension 2. So any vector field $\zeta$ tangent to $R D_{F} \backslash \Delta$ is liftable under $\tilde{F}$ in the vector field $\eta$ defined over $\boldsymbol{C}^{n} \times \boldsymbol{C}^{p} \backslash \Sigma$, where $\Sigma:=\tilde{F}^{-1}(\Delta)$, which is of codimension 2 . So $\eta$ can be uniquely extended to $\boldsymbol{C}^{n} \times \boldsymbol{C}^{p}$.

So the previous proposition tells us that $R D_{G}$ (or $R D_{F}$ ) is a free divisor if any liftable vector field by $\tilde{G}$ belongs to $\mathscr{L}$.

The following proposition shows us that what could be a sufficient condition to have a free divisor structure on the residual discriminant, with liftable vector fields.

Proposition 2.16. Let $H$ be an Euler free divisor and let $f$ be a function of finite relative codimension $p>1$ along $H$. Let $F$ be a mini-versal unfolding of $f$, 
and let $G$ be the truncated mini-versal unfolding of $f$ coming from $F . \quad$ If $\operatorname{dim} R D_{G} \cap$ $\left(H \times C^{p-1}\right)=p-2$. Then $R D_{G}$ is a free divisor and the vector fields tangent to $R D_{G}$ are liftable by $\tilde{G}$ in vector fields tangent to $H \times C^{p-1}$.

Proof. In fact we show that any vector field tangent to $R D_{G}$ is liftable in a vector field tangent to $H \times C^{p-1}$. The proof is exactly the same as that of Proposition 2.15 (ii). So $\Theta_{R D_{G}}=\mathscr{L}$ and then is a free $\mathcal{O}_{y, v}$-module of rank $p$, that is, $R D_{G}$ is a free divisor.

Remark 2.2. 1) If $\operatorname{dim} R C_{F} \cap\left(H \times C^{p}\right)=p-1$, then $\mathcal{O}_{R C_{F}}$ is reduced, since at any point which is not on $H \times C^{p}, \Theta_{H}$ is a basis of the vector fields of the ambient space. By a coordinate change $h$ becomes an invertible coordinate. So the argument of Lemma 2.5 provides a Zariski dense open set of points in $R C_{F}$ at which the above structure is reduced. Thus the genericity criterion for a Cohen-Macauley space of [dPGW, Corollary 1.18] gives the reduced structure everywhere. This will be very important in the next section.

2) Under the hypotheses of the previous proposition, we obtain a free basis of the $\mathcal{O}_{y, v}$-module of the vector fields tangent to the (truncated) residual discriminant $R D_{G}$, in that case, in repeating the proof of Lemma 2.10, because, in constructing the vector fields we want to be a basis of $\Theta_{R D_{G}}$, we are sure that the determinant of the matrix of these vector fields gives a reduced equation of the residual discriminant, since this matrix is the matrix which appears in the resolution of the residual Jacobian module (that obtained for $G$ ) as an $\mathcal{O}_{y, v^{-}}$ module, and so its determinant is a generator of the 0-th Fitting ideal of this resolution.

In order to produce a basis of vector fields tangent to $R D_{F}$, we just repeat what have been done in the smooth case.

The following corollary is an analog of $[\mathrm{Sa} 2,1.5]$. It will be very useful in the next section to prove that the residual bifurcation set is a free divisor.

Corollary 2.17. If $R C_{G} \cap\left(H \times C^{p-1}\right)$ is of dimension $p-2$, then the following sum of $\mathcal{O}_{v}$-modules is direct

$$
\Theta_{y, v}=\Theta_{R D_{G}} \oplus \mathcal{O}_{v}\left\{\frac{\partial}{\partial y}, \frac{\partial}{\partial v_{1}}, \ldots, \frac{\partial}{\partial v_{p-1}}\right\} .
$$

Proof. This is a straightforward computation once we know a basis of the vector fields tangent to $R D_{G}$.

In the case of a function germ $f$ of finite relative codimension along $H$ equal to 1 , there is the following

Proposition 2.18. Let $H$ be an Euler free divisor such that there exists a function $f$ of finite relative codimension 1 along $H$. Let $F$ be a mini-versal unfolding of $f$. Then $R D_{F}$ is a free divisor. 
Proof. Note that $F(x, u)=h^{2}(x)\left(f_{0}(x)+u\right)=h^{2}(x) F_{0}(x, u)$. So the truncated mini-versal unfolding is just $f$ itself. Then in using the mutiplication map by $f$ in $M_{R C_{G}}$ to obtain a generator of the 0 -th Fitting ideal as in Theorem 1.4, then the generator obtained is $y$. So this provides a reduced structure to $R D_{G}$, hence to $R D_{F}$.

COROLlaRy 2.19. Under the previous hypothesis on $H$ and $f$, then

(i) $f_{0}$ is a submersion.

(ii) There is a vector field $X$ in $\Theta_{H}$ such that $X \cdot f=f$.

(iii) Any vector field tangent to $R D_{F}$ is liftable by $\tilde{F}$ in a vector field tangent to $H \times C^{p}$.

Proof. (i) We can suppose that $H$ is Euler and all its tangent vector fields are vanishing at the origin except, possibly, the Euler vector field $E$. Then $\xi_{1} \cdot f_{0}, \ldots, \xi_{n-1} \cdot f_{0}, 2 f_{0}+E \cdot f_{0}$ are generators of the maximal ideal $m_{x}$. Since $\xi_{i}(0)=\left(2 f_{0}+E \cdot f_{0}\right)(0)=0$, this implies that $f_{0} \in m_{x} \backslash m_{x}^{2}$ (otherwise $m_{x}=$ $\left.E \cdot f_{0}+m_{x}^{2}\right)$. $h^{2} f_{0}$.

(ii) Since $f_{0} \in m_{x}$, then there is a vector field $X \in \Theta_{H}$ such that $X \cdot\left(h^{2} f_{0}\right)=$

(iii) By (ii) there is a vector field $X \in \Theta_{H}$ such that $X \cdot f=f$. This means that the vector field $y(\partial / \partial y)$ is liftable by $G=f$ the truncated mini-versal unfolding, and so that the ringed structure over $R D_{G}=\{0\} \subset C$ given by means of the 0 -th Fitting ideal is reduced.

Remark 2.3. The problem we have is not to decide if the (truncated) residual disriminant is a free divisor or not, but to decide if the vector fields tangent at the (truncated) residual discriminant are liftable in vector fields tangent to a suspension of $H$, in which case the (truncated) residual discriminant is a free divisor.

As suggested by the very simple examples (and not quite representative of the general situation) before, it seems that the key element is the way that the truncated residual critical locus intersects the suspension of $H$ (in fact the singular set of $H$ ). We can think that informations about this are contained in the smallest jets at the origin of the vector fields tangent to $H$ which are non identically zero of the vector fields tangent to $H$ (see Damon and his conditions on the genericity of Morse type singularities on the divisor [Da-I] and the genericity of the locally liftable vector fields $[\mathrm{Da}-\mathrm{II}])$.

By now we are not able to find necessary conditions on the divisor $H$ to obtain that for any function germ of finite relative codimension along $H$ in order to be able to lift the vector fields tangent to the (truncated) residual discriminant in vector fields tangent to the suspension of $H$.

\subsection{Bifurcation sets and free divisor}

Since we have proved that the residual discriminant of any function germ of finite relative codimension along an Euler free divisor, and under the hypothesis $R C_{G} \cap\left(H \times C^{p-1}\right)$ is $=p-2$, is a free divisor, it is natural to ask if the residual 
bifurcation set is also a free divisor as it is for an isolated hypersurface singularity ([Bru2], [Ter2] and [Go]).

Let $W_{k}(Y, a)$ be the Weierstrass polynomial of degree $k$, that is, $\operatorname{WP}_{k}(Y, a)=$ $Y^{k}+a_{1} Y^{k-1}+\cdots+a_{k-1} Y+a_{k}$, where the $a_{i}$ 's are in $C$ for $1 \leq i \leq k$. Let us denote by $\Pi_{k}: \boldsymbol{C} \times \boldsymbol{C}^{k} \rightarrow \boldsymbol{C}^{k}$ the projection on the $a_{i}$ 's plane. Let $W \Delta_{k}$ be the zero set (smooth) of $W P_{k}$ in $\boldsymbol{C} \times \boldsymbol{C}^{k}$ and let $W \Sigma_{k} \subset \boldsymbol{C}^{k}$ be the discriminant of $W \Delta_{k}$ which is also the discriminant of $\left.\Pi_{k}\right|_{W \Delta_{k}}$. We recall that $W \Sigma_{k}$ is a free divisor and that its smooth points are the $u$ 's where $W P_{k}$ admits a double root with $k-2$ simple roots. Moreover the logarithmic stratification of $W \Sigma_{k}$ is finite and given by the Samuel stratification (see $[\mathrm{DR}]$ or $[\mathrm{Me}]$ ).

Let $D$ be a free divisor in $\left(\boldsymbol{C}^{p}, 0\right)$ and let $A:\left(\boldsymbol{C} \times \boldsymbol{C}^{p-1}, 0\right) \rightarrow\left(\boldsymbol{C} \times \boldsymbol{C}^{p}, 0\right)$ with $(y, v) \rightarrow A(y, v)=\left(y, a_{1}(v), \ldots, a_{p}(v)\right)$ such that $\delta(y, v)=W P_{p} \circ A(y, v)$ is a reduced equation of $D$. Let us denote $\mathcal{O}_{D}:=\mathcal{O}_{y, v} / \delta \mathcal{O}_{y, v}$. Since the singular set $\Sigma(D)$ of this free divisor is a determinantal space of codimension 2 in $\left(\boldsymbol{C} \times \boldsymbol{C}^{p-1}, 0\right)$ and since $\pi_{D}:=\left.\Pi_{p-1}\right|_{D}$ is a ramified covering of degree $p$ over $\left(\boldsymbol{C}^{p-1}, 0\right)$, then any smooth point of $\pi_{D}(\Sigma(D))$ is a smooth point of the discriminant of $\pi_{D}$ which is $\operatorname{Bif}(D)=\pi_{D}(\{\delta=\partial \delta / \partial y=0\})$.

LEMma 2.20. If $\{\delta=\partial \delta / \partial y=0\}=\Sigma(D)$, then any vector field in $\Theta_{u}$, tangent to $\operatorname{Bif}(D)$, is liftable by $\Pi_{p-1}$ to a vector field tangent to $D$.

Proof. The proof follows in applying [Ly2, Theorem 5] of Lyashko which enables to lift a vector tangent to the bifurcation set (the discriminant of a linear projection) to a vector field tangent to a discriminant, since our hypotheses satisfy those of the quoted result, that is

$$
\operatorname{dim}\left\{\delta=\frac{\partial \delta}{\partial y}=\frac{\partial^{2} \delta}{\partial y^{2}}=0\right\}<\operatorname{dim}\left\{\delta=\frac{\partial \delta}{\partial y}=0\right\} .
$$

Now we follow the steps of the proof of Terao to show that the bifurcation set of an isolated hypersurface singularity is also a free divisor [Ter2]. For this purpose from now we suppose that $\{\delta=\partial \delta / \partial y=0\}=\Sigma(D)$. First we define the $\mathcal{O}_{v}$-module of the $\Pi_{p-1}$-lowerable vector fields that is

$$
\mathscr{K}=\left\{\xi \in \Theta_{y, v}: \xi \cdot v_{i} \in \mathcal{O}_{v} \quad i=1, \ldots, p-1\right\}=\mathcal{O}_{y, v} \frac{\partial}{\partial y}+\mathcal{O}_{v} \Theta_{v} .
$$

Let $\Pi_{D}$ be the following $\mathcal{O}_{v}$-module homomorphism $\left(\pi_{p-1}\right)_{*}: \mathscr{K} \cap \Theta_{D} \rightarrow \Theta_{v}$. Note that the kernel of $\Pi_{D}$ is the submodule of $\Theta_{y, v}$ generated by $\delta(\partial / \partial y)$. Let us denote by $\mathscr{G}$ the $\mathcal{O}_{v}$-submodule of $\Theta_{y, v}$ generated by $\partial / \partial y$ and the $\left(\partial / \partial v_{i}\right)$ 's. We have $\mathscr{G} \subset \mathscr{K}$. Now we can state and proof the following

Lemma 2.21. Suppose that $\Theta_{y, v}=\Theta_{D} \otimes_{\mathcal{O}_{v}} \mathscr{G}$ as $\mathcal{O}_{v}$-module. Then the $\mathcal{O}_{v^{-}}$ module $\left(\Pi_{p-1}\right)_{*}\left(\mathscr{K} \cap \Theta_{D}\right) \subset \Theta_{B i f(D)}$ is free of rank $p-1$.

Proof. As in [Ter2, Lemma 3.5] the key arguments of the proof are the freeness of $\Theta_{D}$ and the $\mathcal{O}_{v}$ direct sum in hypothesis. 
The natural map $\mathscr{K} \cap \Theta_{D} \rightarrow \mathscr{K} / \mathscr{G}$ is an $\mathcal{O}_{v}$-isomorphism and since $\mathscr{K} / \mathscr{G} \cong$ $\mathcal{O}_{y, v} / \mathcal{O}_{v}$, we have the following diagram

$$
\begin{gathered}
0 \longrightarrow \mathcal{O}_{y, v}\left(\delta_{D} \frac{\partial}{\partial y}\right) \longrightarrow \mathscr{K} \cap \Theta_{D} \stackrel{\Pi_{D}}{\longrightarrow} \mathfrak{T}\left(\Pi_{D}\right) \longrightarrow 0 \\
0 \longrightarrow \delta_{D} \mathcal{O}_{y, v} \longrightarrow \frac{\mathcal{O}_{y, v}}{\mathcal{O}_{v}} \longrightarrow \frac{\mathcal{O}_{D}}{\mathcal{O}_{v}} \longrightarrow 0 .
\end{gathered}
$$

The isomorphism between $\mathscr{K} \cap \Theta_{D}$ and $\mathcal{O}_{y, v} / \mathcal{O}_{v}$ is given by $\xi \rightarrow[\xi \cdot y]$ (the bracket meaning the residue class). So we have an $\mathcal{O}_{v}$-isomorphism

$$
\begin{aligned}
\alpha: \operatorname{Im}(\Pi) & \rightarrow \frac{\mathcal{O}_{D}}{\mathcal{O}_{v}} \\
\Pi(\xi) & \rightarrow \xi \cdot y \bmod \mathcal{O}_{v} .
\end{aligned}
$$

Since $\delta$ is reduced and $\pi_{D}$ finite, as $C$ local algebras we have

$$
\frac{\mathcal{O}_{D}}{\mathcal{O}_{v}} \stackrel{\sim}{\rightarrow} \frac{C\{y\}}{\left(y^{p}\right)} .
$$

And by Nakayama's Lemma $[1],[y], \ldots,\left[y^{p-1}\right]$ generate freely the $\mathcal{O}_{u}$-module $\mathcal{O}_{D}$. So $\mathcal{O}_{D} / \mathcal{O}_{v}$ is freely generated over $\mathcal{O}_{v}$ by $[y], \ldots,\left[y^{p-1}\right]$, which ends the proof.

THEOREM 2.22. Bif $(D)$ is a free divisor.

Proof. This is immediate since any vector fields $\eta$ which is tangent to the bifurcation set is liftable under $\Pi_{p-1}$ in a vector field tangent to $D$. Then by the last lemma we conclude.

Let $D=R D_{G}$ be the residual discriminant of a truncated mini-versal unfolding $G$ of a function germ $f$ singular along an Euler free divisor $H \subset\left(\boldsymbol{C}^{n}, 0\right)$ whose reduced ideal is $I$. Let $p$ be the dimension of $I^{2} / T_{e} \mathscr{R}_{I}(f)$. By now, we suppose that the dimension of $R C_{G} \cap\left(H \times C^{p-1}\right)$ is equal to $p-2$. So the dimension of $R C_{F} \cap\left(H \times C^{p}\right)$ is equal to $p-1$. We have the following

Proposition 2.23. Let $\delta_{F}(y, u)$ be the reduced equation of $R D_{F}$, provided by the determinant of the matrix whose columns are the coefficients of the previous basis of the vector fields tangent to $R D_{F}$. Then $\left\{\delta_{F}=\partial \delta_{F} / \partial y=0\right\}=\Sigma\left(R D_{F}\right)$.

Proof. Let $\tilde{\mathcal{O}}$ be the sheaf of holomorphic function germ on a small neighbourhood $\Omega$ of the origin of $\boldsymbol{C}^{n} \times \boldsymbol{C}^{p}$. Let $\mathscr{W}=\Omega \backslash H \times \boldsymbol{C}^{p}$ and let $\mathscr{V}=\pi_{p}(\boldsymbol{\Omega})$.

We recall that the $\tilde{\mathcal{O}}$-module sheaves $\mathscr{M}:=\mathscr{M}_{R C_{F}}$ and $\mathscr{N}:=\tilde{\mathscr{O}}_{\Sigma(F)}$ are equal on $\mathscr{W}$. Since $M_{R C_{F}}:=\mathscr{N}_{(0,0)}$ is $\mathcal{O}_{u}$-free with basis $e_{1}, \ldots, e_{p}$, we can suppose that $\Omega$ is small enough to have representatives of the $e_{i}=\left(\partial F / \partial u_{i}\right)$ 's over $\Omega$.

The Malgrange-Weierstrass Preparation Theorem insures us that for any $u_{0} \in \mathscr{W}$ the complex vector space $\mathscr{N} /\left(U_{1}, \ldots, U_{p}\right)$ is a finite dimensional $\left(\left(U_{1}, \ldots\right.\right.$, 
$\left.U_{p}\right)$ is a system of local coordinates at $u_{0}$ in $\boldsymbol{C}^{p}$ ), and is generated by $e_{1}, \ldots, e_{p}$. This means that $F$ is a versal unfolding of the function germ $f_{u_{0}}$ with an isolated singularity at $x_{0}$. The Morse points form a Zariski open dense subset of any neighbourhood of $\left(x_{0}, u_{0}\right) \in R C_{F} \cap \mathscr{W}$. Since $R C_{F} \cap H \times C^{p}$ is of codimension 1 in $R C_{F}$, this shows that the subset of $R C_{F}$ of the $\left(x_{0}, u_{0}\right)$ such that $f_{u_{0}}$ is a Morse function is open and dense in $R C_{F}$.

Now we use an argument similar to [Tei, Section 5.5] to control, on the residual dicriminant, the vanishing locus of the partial derivatives of $\delta_{F}$ in the directions of the unfolding parameters by the vanishing of the partial derivative of $\delta_{F}$ along the $y$-axis.

Note that $\left.\delta_{F} \circ \tilde{F}\right|_{R C_{F}} \equiv 0$. By the reduced structure of $R C_{F}$, we obtain that

$$
\delta_{F} \circ \tilde{F}(x, u)=\sum_{i=1}^{n} \frac{a_{i}(x, u)}{h^{2}(x)} \xi_{i}(x) \cdot F(x, u) .
$$

Thus we obtain that

$$
\begin{aligned}
& 0=\frac{\partial\left(\delta_{F} \circ \tilde{F}\right)}{\partial u_{j}}=\sum_{i=1}^{n} \frac{a_{i}}{h^{2}} \frac{\partial\left(\xi_{i} \cdot F\right)}{\partial u_{j}} \bmod \tilde{T}_{e} \mathscr{R}_{I}(f), \\
& 0=\xi_{j} \cdot\left(\delta_{F} \circ \tilde{F}\right)=\sum_{i=1}^{n} \frac{a_{i}}{h^{2}} \xi_{j} \cdot\left(\xi_{i} \cdot F\right) \bmod \tilde{T}_{e} \mathscr{R}_{I}(f) .
\end{aligned}
$$

We easily see that the $n \times n$-matrix $\left[\xi \cdot\left(\xi_{j} \cdot F\right)\right]$ is conjugated to the Hessian matrix of $f_{u_{0}}$, and so, we deduce that $a_{i}$ is vanishing over $R C_{F} \cap \mathscr{W}$. Since $R C_{F}$ is endowed with a reduced Cohen-Macauley ringed structure it is equidimensional and $R C_{F} \cap \mathscr{W}$ is an open dense subset of $R C_{F}$. Then $a_{i}$ vanishes over $R C_{F}$, which means that $a_{i}$ belongs to $\tilde{T}_{e} \mathscr{R}_{I}(f)$. So we obtain

$$
0=\left.\frac{\partial\left(\delta_{F} \circ \tilde{F}\right)}{\partial u_{j}}\right|_{R C_{F}}=\left.\left.\frac{\partial \delta_{F}}{\partial y} \circ \tilde{F}\right|_{R C_{F}} \cdot \frac{\partial F}{\partial u_{j}}\right|_{R C_{F}}+\left.\frac{\partial \delta_{F}}{\partial u_{j}} \circ \tilde{F}\right|_{R C_{F}} .
$$

Since $R D_{F}$ is by definition $\tilde{F}\left(R C_{F}\right)$ the above equality proves that

$$
\left\{\delta_{F}=\frac{\partial \delta_{F}}{\partial y}=\frac{\partial \delta_{F}}{\partial u_{1}}=\cdots=\frac{\partial \delta_{F}}{\partial u_{p}}=0\right\}=\left\{\delta_{F}=\frac{\partial \delta_{F}}{\partial y}=0\right\}
$$

which is the desired result.

Let $G$ be the truncated mini-versal unfolding of $f$. Since $\Phi\left(R D_{F}\right)=R D_{G} \times C$ with $\Phi\left(y, v, u_{p}\right)=\left(\phi_{0}(y, u), v, u_{p}\right)$, we have $\Phi\left(\Sigma\left(R D_{F}\right)\right)=\Sigma\left(R D_{G}\right) \times C$. We also have the following commutative diagrams

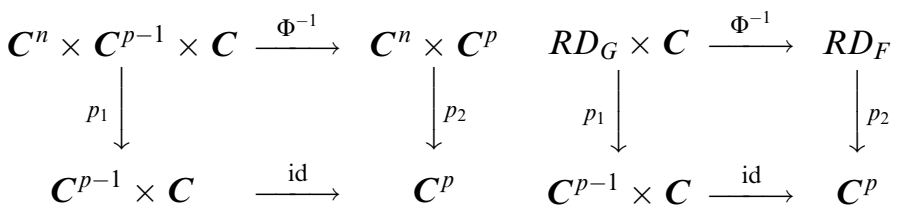


where $p_{1}$ and $p_{2}$ are the obvious linear projections on $\boldsymbol{C}^{p}=\boldsymbol{C}^{p-1} \times \boldsymbol{C}$. Note that the form of $\Phi$ gives us that $R B_{G} \times C=R B_{F}$. From this we deduce that $\left.p_{1}\right|_{R D_{G} \times C}$ is a diffeomorphism at the point $(y, u)=\left(y, v, u_{p}\right)$ if and only if $\left.p_{2} \circ \Phi^{-1}\right|_{R D_{F}}$ is also a diffeomorphism at the point $\Phi^{-1}\left(y, v, u_{p}\right)=\left(\left(\Phi^{-1}\right)_{0}, v, u_{p}\right)$. This means that the critical set of $p_{1}$ is exactly $\Sigma\left(R D_{G}\right)$. So by Theorem 2.22 $\operatorname{Bif}\left(R D_{G}\right)=R B_{G}$ is a free divisor. So we have proved the following

THEOREM 2.24. Let $f$ be a function germ of finite codimension $p$ relatively to an Euler free divisor $H$. Let $f$ be its mini-versal unfolding. Then the residual bifurcation set of $F$ is a free divisor.

To finish, as Terao in [Ter2] and Bruce in [Bru2], we can describe a basis of the vector fields tangent to $R B_{F}=\operatorname{Bif}\left(R D_{F}\right)$. The proof of that kind of result is now well known (see also [Go, Section 4$]$ ). We begin by producing some vector fields tangent to $R B_{G}$.

For any $j=1, \ldots, p-1$ we have in $\mathcal{O}_{x, v}$

$$
-G^{j} h^{2}=G^{j} h^{2} e_{p}=\sum_{i=1}^{n-1} \gamma_{i, j} \xi_{j} \cdot G+\gamma_{j} E_{(h)} \cdot G+h(x)\left[\sum_{i=1}^{p-1} c_{i, j}(v) e_{j}+c_{j}(v) e_{p}\right] .
$$

Now we define the vector fields $\mu_{j}$ in $\left(\boldsymbol{C}^{p-1}, 0\right), j=1, \ldots, p-1$

$$
\mu_{j}=\sum_{i=1}^{p-1} b_{i, j}(v) \frac{\partial}{\partial v_{i}} .
$$
$\Theta_{R B_{G}}$.

THEOREM 2.25. The vector fields $\mu_{j}$, for $j=1, \ldots, p-1$, form a free basis of

Proof. We build the vector fields $\phi_{1}, \ldots, \phi_{p-1} \in \mathscr{K} \cap \Theta_{R D_{G}}$ such that $\Pi_{R D_{G}}\left(\phi_{1}\right), \ldots, \Pi_{R D_{G}}\left(\phi_{p-1}\right)$ form a free basis of $\Theta_{R D_{G}}$ if and only if $\left[\phi_{1} \cdot y\right], \ldots$, $\left[\phi_{p-1} \cdot y\right]$ is an $\mathcal{O}_{v}$-free basis of $\mathcal{O}_{D} / \mathcal{O}_{v}$ (see [Ter2, Theorem C]). Now we use the basis of $\Theta_{R D_{G}}$ given previously to define inductively $\phi_{i}(1 \leq i \leq p-1)$ by

$$
\phi_{1}=\eta_{p} \quad \text { and } \quad \phi_{i}=y \phi_{i-1}+\sum_{j=1}^{p-1}\left(\phi_{i-1} \cdot v_{j}\right) \eta_{j} .
$$

Thus for any $i=1, \ldots, p-1 \phi_{i}$ is tangent to $R D_{G}$. For $k=1, \ldots, p-1$ we obtain

$$
\begin{aligned}
\phi_{i} \cdot v_{k} & =y \phi_{i-1} \cdot v_{k}+\sum_{j=1}^{p-1}\left(\phi_{i-1} \cdot v_{j}\right) \eta_{j} \cdot v_{k} \\
& =y \phi_{i-1} \cdot v_{k}+\left(\phi_{i-1} \cdot v_{k}\right)\left(a_{k, k}-y\right)+\sum_{j \neq t}^{p}\left(\phi_{i-1} \cdot v_{j}\right) a_{k, j} \\
& =\sum_{j=1}^{p-1} a_{k, j}\left(\phi_{i-1} \cdot v_{j}\right),
\end{aligned}
$$


and this proves that $\phi_{i} \in \mathscr{K} \cap \Theta_{R D_{G}}$ and that $\Pi_{R D_{G}}\left(\phi_{i}\right)=\mu_{i}$. To finish, we have

$$
\phi_{i} \cdot y=y\left(\phi_{i-1} \cdot y\right)+\sum_{j=1}^{p-1}\left(\phi_{i-1} \cdot v_{j}\right)\left(-a_{j, p}\right)=y\left(\phi_{i-1} \cdot y\right) \bmod \mathcal{O}_{v} .
$$

Thus we find that $\left[\phi_{i} \cdot y\right]=\left[y^{i-2} \phi_{1} \cdot y\right]=\left[y^{i-2}\left(y-a_{p, p}(v)\right)\right] \in \mathcal{O}_{R D_{G}} / \mathcal{O}_{v}$ and thus $\left[\phi_{1} \cdot y\right], \ldots,\left[\phi_{p-1} \cdot y\right]$ is a free $\mathcal{O}_{v}$-generating family of $\Theta_{R B_{G}}$.

COROLlaRy 2.26. $\operatorname{det}\left|\mu_{1}, \ldots, \mu_{p-1}\right|$ is a reduced equation of $R B_{G}$.

Proof. Since $\mu_{1}, \ldots, \mu_{p-1}$ form a free basis of $R B_{G}$, then the determinant of the matrix of their coefficients is only vanishing over $R B_{G}$. By Saito's Lemma ([Sa1, Lemma 1.9]) we conclude.

COROllary 2.27. The vector fields $\mu_{j}$, for $j=1, \ldots, p-1$, and $\partial / \partial u_{p}$ form a free basis of $\Theta_{R B_{F}}$.

\subsection{Comments on the non-Euler free divisor case}

Let $H \subset\left(\boldsymbol{C}^{n}, 0\right)$ be a free divisor which is not necessarily Euler and given by a reduced equation $h \in \mathcal{O}_{x}$. To produce an Euler free divisor from $H$ with the same geometry, there is just to apply the trick of the good defining equation (see [DM, Section 3]), that is, we look at $H$ in $\boldsymbol{C}^{n} \times \boldsymbol{C}$ (as a source space with coordinates $(x, w))$ as the hypersurface $\tilde{H}=H \times C$ which is now Euler and free since

$$
\tilde{H}=\left\{\tilde{h}(x, w)=e^{w} h(x)=0\right\} \quad \text { and } \quad \Theta_{\tilde{H}}=\left(\Theta_{H} \otimes_{\mathcal{O}_{x}} \mathcal{O}_{x, w}\right) \oplus \mathcal{O}_{x, w} \frac{\partial}{\partial w} .
$$

Such an $\tilde{h} \in \mathcal{O}_{x, w}$ provides a reduced equation of $H \times \boldsymbol{C}$ and is called a good defining equation.

We would like to know if, given a non-Euler free divisor $H$ and a function of finite codimension relatively to $H$, say $f$, there is a way to find a function $g$ associated with $f$ which will be of finite codimension relatively to $\tilde{H}$ and which will provide a residual discriminant obtained as a one-dimensional fibration along that of $f$. In the case of isolated hypersurface singularities such a notion exists, which is the stably equivalence in the terminology of Arnol'd.

Let $f=h^{2}(x) f_{0}(x)$ be of finite codimension relatively to $H$ and let $k(x, w)=$ $e^{2 w} h(x)^{2} k_{0}(x, w)$ with $k_{0}(x, w)=f_{0}(x)+w^{2}$. From the point of view of the isolated singularities along a variety the functions $f_{0}$ and $k_{0}$ are stably equivalent. The $\mathcal{O}_{x, w}$-module $\Theta_{\tilde{h}}^{(0)}$ defined as the submodule of the vector fields tangent to the levels of $\tilde{h}$ is freely generated by $\chi_{i}(x, w)=\xi_{i}(x)-2 a_{i}(x)(\partial / \partial w)$ for $i=1, \ldots, n$. So the vector fields $\partial / \partial w$ and the $\chi_{i}$ 's form an $\mathcal{O}_{x, w}$-free basis of the vector fields tangent to $\tilde{H}$. 
Since

$$
\begin{aligned}
\frac{(h)^{2}}{T_{e} \mathscr{R}_{(h)}(f)} \cong \frac{\mathcal{O}_{x, w}}{\left(\xi_{i} \cdot f_{0}+2 a_{i} f_{0}, w\right)}, \\
\frac{(\tilde{h})^{2}}{T_{e} \mathscr{R}_{(\tilde{h})}(k)} \cong \frac{\mathcal{O}_{x, w}}{\left(\xi_{i} \cdot k_{0}+2 a_{i} k_{0}, k_{0}+w\right)} .
\end{aligned}
$$

Note that the following ideals in $\mathcal{O}_{x, w},\left(T_{e} \mathscr{K}_{(h)}, w\right)=\left(f_{0}, \xi_{i} \cdot f_{0}+2 a_{i} f_{0}, w\right)$ and $T_{e} \mathscr{K}_{(\tilde{h})}=\left(k_{0}, \xi_{i} \cdot k_{0}+2 a_{i} k_{0}, k_{0}+w\right)$ are equal. But we do not know if the above local $\boldsymbol{C}$-algebras are isomorphic or not.

\section{Counter-example when $H$ is a smooth space of codimension 2}

Let $I=\left(x_{1}, x_{2}\right)$. Then $\Theta_{I, e}$, the $\mathcal{O}_{x}$-module of the vector fields tangent to $I$, is generated by $\partial / \partial x_{3}, \ldots, \partial / \partial x_{n}$, and $x_{1}\left(\partial / \partial x_{1}\right), x_{2}\left(\partial / \partial x_{1}\right), x_{1}\left(\partial / \partial x_{2}\right), x_{2}\left(\partial / \partial x_{2}\right)$. In his papers ([Za1] and [Za2]) Zaharia gives some normal forms of the simple nonisolated singularities along a smooth space of codimension 2 . We give just below two of these classes where the respective residual critical loci are rather different. Let $z_{i}=x_{i}$ for $i=1,2$ and $w_{j}=x_{j+2}$ for $j=1, \ldots, n-2$.

The first case is given by the normal form $I I A_{s}: f(z, w)=w_{1} z_{1}^{2}+w_{2} z_{2}^{2}+$ $z_{1} z_{2} w_{3}^{s+1}$. The family $\left\{z_{1} z_{2} w_{3}^{k}\right\}_{\{k=0, \ldots, s-1\}}$ is a $\boldsymbol{C}$-basis of the vector space $I^{2} / T_{e} \mathscr{R}_{I}(f)$, and thus $F(x, u)=w_{1} z_{1}^{2}+w_{2} z_{2}^{2}+z_{1} z_{2}\left(w_{3}^{s+1}+u_{1} w_{3}^{s-1}+\cdots+u_{s}\right)$. Let us denote $\left(w_{3}^{s+1}+u_{1} w_{3}^{s-1}+\cdots+u_{s}\right)$ by $Q_{u}\left(w_{3}\right)$. After computations we obtain that

$$
\begin{aligned}
& \tilde{T}_{e} \mathscr{R}_{I}(f)=\left\{z_{1}^{2}, z_{2}^{2}, z_{1} z_{2} Q_{u}\left(w_{3}\right), z_{1} z_{2} Q_{u}^{\prime}\left(w_{3}\right), z_{1} z_{2} w_{i} i \neq 3\right\}, \\
& \tilde{T}_{e} \mathscr{R}_{I}(f) \cap\left(z_{1} z_{2}\right)=\left(z_{1} z_{2}\right)\left(z_{1}, z_{2}, Q_{u}\left(w_{3}\right), 2 w_{i} i \neq 3\right) .
\end{aligned}
$$

We see that among the generators $z_{1} z_{2}, z_{1} z_{2} w_{3}, \ldots, z_{1} z_{2} w_{3}^{s-1}$ of $M_{R C_{F}}$ there is an $\mathcal{O}_{u}$-relation whereas any subfamily of $(s-1)$ elements is $\mathcal{O}_{u}$-free:

$$
\begin{aligned}
z_{1} z_{2}\left[(s+1) Q_{u}-x_{3} Q_{u}^{\prime}\right] & =z_{1} z_{2}\left[2 u_{1} w_{3}^{s-1}+3 u_{2} w_{3}^{s-2}+\cdots+(s+1) u_{s}\right] \\
& =0 \bmod \tilde{T}_{e} \mathscr{R}_{I}(f) .
\end{aligned}
$$

Thus $R C_{F}=\left\{z_{1}=z_{2}=Q_{u}\left(w_{3}\right)=Q_{u}^{\prime}\left(w_{3}\right)=w_{1}=w_{2}=0\right\}$ and it is a complete intersection of dimension $s-1$, so the residual discriminant $R D_{F}$ is necessarily of codimension at least 2 in $\left(\boldsymbol{C} \times \boldsymbol{C}^{s}, 0\right)$. We can verify that the residual discriminant of $I I A_{s}$ is actually the discriminant of $A_{s}$ embedded in $0 \times C^{s}$. We can also verify that in the classes $I I A-D-E([\mathrm{Za} 2])$ there is always an $\mathcal{O}_{u}$-relation between the $\mathcal{O}_{u}$ generators of $M_{R C_{F}}$, but every subfamily is $\mathcal{O}_{u}$-free. The residual critical locus is still a complete intersection of dimension $s-1$ contained in $H$, and so the corresponding residual discriminant is in $0 \times \boldsymbol{C}^{s}$.

These facts are quite general since we have by staightforward calculus $([\mathrm{Gr}])$ the following 
Proposition 3.1. Let $f(z, w)=z_{1}^{2} w_{1}+z_{2}^{2} w_{2}+z_{1} z_{2} g(z, w)$, with $g$ such that $\left(\partial g / \partial w_{1}\right)(0)\left(\partial g / \partial w_{2}\right)(0) \neq 1$. Then $f$ is finitely determined relatively to $H=$ $\left\{z_{1}=z_{2}=0\right\}$ if and only if $g_{0}$ the restriction of $g$ to $\left\{z_{1}=z_{2}=w_{1}=w_{2}=0\right\}$, has an isolated singularity at the origin of $\left(C^{n-4}, 0\right)$.

Proposition 3.2. Let $f$ be a finitely determined germ relatively to the smooth space $H=\left\{z_{1}=z_{2}=0\right\}$ and $f$ is of the form $z_{1}^{2} w_{1}+z_{2}^{2} w_{2}+z_{1} z_{2} g(z, w)$. When $\left(\partial g / \partial w_{1}\right)(0)\left(\partial g / \partial w_{2}\right)(0) \neq 1$ then $s-1$.

(i) $R C_{F}$ is a smooth complete intersection of dimension $\operatorname{dim}_{C}\left(I^{2} / T_{e} \mathscr{R}_{I}(f)\right)-1=$

(ii) $R D_{F}=0 \times \Delta\left(g_{0}\right) \subset 0 \times C^{s}$, where $\Delta\left(g_{0}\right)$ is the discriminant of the isolated hypersurface singularity $g_{0}$.

It is easy to show that for any function germ singular along $H=\left\{z_{1}=\right.$ $\left.z_{2}=0\right\}$ and of finite relative codimension which can be written as $f(z, w)=$ $z_{1}^{2} w_{1}+z_{2}^{2} w_{2}+z_{1} z_{2} g(z, w)$, then (in passing to jets) the condition $\left(\partial g / \partial w_{1}\right)(0)$. $\left(\partial g / \partial w_{2}\right)(0) \neq 1$ is a generic condition for such function germs.

The next normal form is $I I B_{s}: f(z, w)=w_{2} z_{1} z_{2}+w_{1} z_{1}^{2}+z_{2}^{2}\left(z_{2}+w_{1}^{s}\right)$. Then $z_{2}^{2}, z_{2}^{2} w_{1}, \ldots, z_{2}^{2} w_{1}^{s-1}$ is a $\boldsymbol{C}$-basis of $I^{2} / T_{e} \mathscr{R}_{I}(f)$ and thus $F(x, u)=w_{2} z_{1} z_{2}+$ $w_{1} z_{1}^{2}+z_{2}^{2}\left(z_{2}+w_{1}^{s}+u_{1} w_{1}^{s-1}+\cdots+u_{s}\right)$. Let us denote $\left(w_{1}^{s}+u_{1} w_{1}^{s-1}+\cdots+u_{s}\right)$ by $Q_{u}\left(w_{1}\right)$. A system of generators for $\tilde{T}_{e} \mathscr{R}_{I}(f)$ are $z_{1} z_{2}, z_{1}^{2}+z_{2}^{2} Q_{u}^{\prime}\left(w_{1}\right)$, $z_{2}^{2}\left(3 z_{2}+2 Q_{u}\left(w_{1}\right)\right), w_{2} z_{2}^{2}$ and $z_{1}^{2} w_{1}$. We find that the residual critical locus is

$$
\begin{aligned}
R C_{F}= & \left\{z_{1}=w_{2}=z_{2} Q_{u}^{\prime}\left(w_{1}\right)=3 z_{2}+2 Q_{u}\left(w_{1}\right)=w_{1} Q_{u}^{\prime}\left(w_{1}\right) 0\right\} \\
= & \left\{z_{1}=w_{2}=Q_{u}^{\prime}\left(w_{1}\right)=3 z_{2}+2 Q_{u}\left(w_{1}\right)=0\right\} \cup\left\{z_{1}=w_{2}=z_{2}\right. \\
& \left.=w_{1}=u_{s}=0\right\}=C_{R, 1} \cup C_{R, 2} .
\end{aligned}
$$

Note that $C_{R, 1}$ is a graph of codimension 4 and not contained in $H$, while $C_{R, 2}$ is smooth of codimension 5 and so contained in $H$ but not in $C_{R, 1}$ (then $M_{R C_{F}}$ cannot be a free $\mathcal{O}_{u}$-module). Note that we have $\Sigma_{R}(\tilde{F})=C_{R, 1}$. That kind of result is also quite general for function germs of finite relative codimension which can be written as $f(z, w)=w_{2} z_{1} z_{2}+w_{1} z_{1}^{2}+z_{2}^{2} g(z, w)$ and with the condition $\left(\partial g / \partial w_{1}\right)(0)+\left(\left(\partial g / \partial w_{2}\right)(0)\right)^{2}=0$ (otherwise they can be written in the generic form above by means of a change of coordinates which preserves the origin and $H)$. In that case the residual critical locus $R C_{F}$ is the union of two distinct complete intersections $C_{R, 1}$ and $C_{R, 2}$ of respective dimensions $\operatorname{dim}_{C}\left(I^{2} / T_{e} \mathscr{R}_{I}(f)\right)$ and $\operatorname{dim}_{C}\left(I^{2} / T_{e} \mathscr{R}_{I}(f)\right)-1$. In that case with the notations of the above propositions $g_{0}$ is still a function with an isolated singularity (which was expected in looking at the above generic function germs), but this is not enough at all to have $f$ of finite relative codimension (for a bit more see [Gr]).

We have some open questions about this last case. For instance we do not know if the hypersurface $\tilde{F}\left(C_{R, 1}\right)$ is a free divisor. What are necessary and sufficient conditions on $g$ for $f$ of the form $w_{2} z_{1} z_{2}+w_{1} z_{1}^{2}+z_{2}^{2} g(z, w)$ to be of finite relative codimension ? Do the residual discriminant of any such function $f$ of 
finite relative codimension control, by its partition into logarithmic strata, the analytic types of the germs obtained by deformation of $f$ ? (A positive answer to this last question could explain the impossible adjacencies occuring in the list given by Zaharia $[\mathrm{Za} 2])$.

\section{REFERENCES}

[Al1] A. G. Aleksandrov, Milnor number of non isolated Saito singularities, Funct. Anal. Appl., 21 (1987), 1-9.

[A12] A. G. Aleksandrov, Non isolated Saito singularities, Math. USSR-Sb., 65 (1990), 561574.

[Ar] V. I. ARNOL'D, Critical points of functions on a manifold with boundary, the simple Lie groups $B_{k}, C_{k}$ and $F_{4}$ and singularities of evolutes, Russian Math. Surveys, 33-5 (1978), 99-116.

[Brul] J. W. Bruce, Functions on discriminants, J. London Math. Soc., 30 (1984), 551-567.

[Bru2] J. W. BRUCE, Vector fields on discriminant and bifurcation varieties, Bull. London Math. Soc., 17 (1985), 257-262.

[BdPW] J. W. Bruce, A. A. Du Plessis and L. C. Wilson, Discriminants and liftable vector fields, J. Algebraic Geom., 3 (1994), 725-753.

[BR] J. W. BRuCE AND R. M. Roberts, Critical points on analytic varieties, Topology, 27 (1988), 57-90.

[Da-I] J. Damon, On the legacy of free divisor: discriminants and Morse-type singularities, Amer. J. Math., 120 (1998), 453-492.

[Da-II] J. Damon, On the legacy of free divisor II: free* divisors and complete intersections, Preprint. (available at http://www.math.unc.edu/Faculty/jndamon)

[Da-III] J. Damon, On the legacy of free divisor III: functions and divisors on complete intersections, Preprint. (available at http://www.math.unc.edu/Faculty/jndamon)

[DM] J. DAmon AND D. Mond, $\mathscr{A}$-Codimension and the vanishing topology of discriminants, Invent. Math., 106 (1991), 217-242.

[Di] A. Dimca, Functions germs defined on isolated hypersurface singularities, Compositio Math., 53 (1984), 245-258.

[DR] A. Dimca AND R. Rosian, The Samuel stratification of the discriminant is Whitney regular, Geom. Dedicata, 17 (1984), 181-184.

[dPGW] A. A. DU Plessis, T. Gaffney and L. C. Wilson, Map-germs determined by their discriminants, Stratifications, Singularities and Differential Equations I (D. Trotman and L. Wilson eds.), Travaux en Cours 54, Hermann, Paris, 1997, 1-40.

[Go] G. Goryunov, Projections and vector fields tangent to the discriminant of a complete intersection, Functional Anal. Appl., 22 (1988), 104-113.

[Gr] V. GRANDJEAn, Stratification logarithmique, détermination finie relative et dicriminant résiduel, Thése, Université de Rennes I, 2000.

[Gu R. Gunning, Lectures on Complex Analytic Varieties: Finite Analytic Mappings, Math. Notes 14, Princeton University Press, Princeton, 1974.

[Ji] G. JIANG, Functions with non isolated singularities on singular spaces, Thesis, Universiteit Utrecht, 1998.

[Lo] E. LooIJenga, Isolated Singular Points on Complete Intersections, London Math. Soc. Lecture Note Ser. 77, Cambridge Univ. Press, Cambridge, 1984.

[Ly1] O. V. Lyashko, Classifications of critical points of functions on a manifold with a boundary, Functional Anal. Appl., 17 (1988), 187-193. 
[Ly2] O. V. Lyashko, Geometry of bifurcation diagrams, J. Soviet. Math., 27 (1984), 27362759 .

[Me] I. Meguerditchian, Du discriminant réel et des polynômes hyperboliques, Thèse, Université de Rennes I, 1991.

[Pe1] R. Pellikane, Finite determinacy of functions with non-isolated singularities, Proc. London. Math. Soc., 57 (1988), 357-382.

[Pe2] R. Pellikan, Deformations of hypersurfaces with one-dimensional singularlocus, J. Pure Appl. Algebra, 67 (1990), 49-71.

[Sa1] K. SAIto, Theory of logarithmic differential forms and logarithmic vector fields, J. Fac. Sci. Univ. Tokyo Sect. 1A Math., 27 (1980), 775-792.

[Sa2] K. SaITo, Primitive form for an unfolding of a function with an isolated critical point, J. Fac. Sci. Univ. Tokyo Sect. 1A Math., 28 (1982), 265-291.

[Ter1] H. Terao, Discriminant of a holomorphic map and logarithmic vector fields, J. Fac. Sci. Univ. Tokyo Sect. 1A Math., 30 (1983), 379-391.

[Ter2] H. Terao, The bifurcation set and logarithmic vector fields, Math. Ann., 263 (1983), 313-321.

[Tei] B. TeIssIER, The hunting of invariants the geometry of the discriminants, Real and Complex Singularities, Oslo 1976 (P. Holm ed.), Sijthoff and Noordhoff, Alphen aan den Rijn, 1977, 565-678.

[Ti] M. Tibar, A note on simple functions, Rev. Roumaine Math. Pures Appl., 37 (1992), $345-352$.

[To] J. C. Tougeron, Idéaux de Fonctions Différentiables, Ergeb. Math. Grenzgeb. (2), 71, Springer, Berlin, 1972.

[Wi] K. Wirthmuller, Singularities determined by their discriminant, Math. Ann., 252 (1965), 237-245.

[Za1] A. ZAHARIA, On simple germs with non-isolated singularities, Math. Scand., 68 (1991), 187-192.

[Za2] A. ZAHARIA, On the bifurcation variety of some non-isolated singularities, Math. Scand., 77 (1995), 45-59.

Laboratoire De MathÉMATiQue

UFR SFA

CAmpus SCIENTIFique

73376 Le Bourget-du-Lac, Cedex, FRANCE

e-mail: Vincent.Grandjean@univ-savoie.fr 